Proceedings of the Edinburgh Mathematical Society (2002) 45, 301-325 (C)

DOI:10.1017/S0013091500001231 Printed in the United Kingdom

\title{
ORNSTEIN-UHLENBECK PROCESSES IN BANACH SPACES AND THEIR SPECTRAL REPRESENTATIONS
}

\author{
JAMES S. GROVES \\ Department of Mathematics and Statistics, University of Lancaster, \\ Lancaster LA1 4YF, UK (j.groves@lancaster.ac.uk)
}

(Received 14 December 2000)

\begin{abstract}
For $Q$ the variance of some centred Gaussian random vector in a separable Banach space it is shown that, necessarily, $Q$ factors through $\ell^{2}$ as a product of 2 -summing operators. This factorization condition is sufficient when the Banach space is of Gaussian type 2. The stochastic integral of a deterministic family of operators with respect to a $Q$-Wiener process is shown to exist under a continuity condition involving the 2 -summing norm. A Langevin equation
\end{abstract}

$$
\mathrm{d} \boldsymbol{Z}_{t}+\Lambda \boldsymbol{Z}_{t} \mathrm{~d} t=\mathrm{d} \boldsymbol{B}_{t},
$$

with values in a separable Banach space, is studied. The operator $\Lambda$ is closed and densely defined. A weak solution $\left(\boldsymbol{Z}_{t}, \boldsymbol{B}_{t}\right)$, where $\boldsymbol{Z}_{t}$ is centred, Gaussian and stationary, while $\boldsymbol{B}_{t}$ is a $Q$-Wiener process, is given when i $\Lambda$ and i $\Lambda^{*}$ generate $C_{0}$ groups and the resolvent of $\Lambda$ is uniformly bounded on the imaginary axis. Both $\boldsymbol{Z}_{t}$ and $\boldsymbol{B}_{t}$ are stochastic integrals with respect to a spectral $Q$-Wiener process.

Keywords: Ornstein-Uhlenbeck processes; Banach spaces; absolutely summing operators; operator groups

AMS 2000 Mathematics subject classification: Primary 60G15

Secondary 46E40; 47B10; 47D03; 60H10

\section{Introduction}

This paper will study a Langevin equation for stochastic processes with values in a separable complex Banach space $E$. The one-dimensional Langevin equation is the Itô differential equation

$$
\mathrm{d} Z_{t}+\rho Z_{t} \mathrm{~d} t=\mathrm{d} b_{t}
$$

for $t \in \mathbb{R}$, where the constant $\rho>0$ describes a frictional resistance. We seek a pair of processes $\left(b_{t}, Z_{t}\right)$, defined on a probability space $(\Omega, \mathcal{F}, \mathbb{P})$, which solve equation (1.1); the process $\left(b_{t}\right)_{t \in \mathbb{R}}$ is required to be a complex Brownian motion on the line with $b_{0}=0$ and the process $\left(Z_{t}\right)_{t \in \mathbb{R}}$ is required to be a complex-valued centred Gaussian stationary stochastic process which is adapted to the filtration induced by $b_{t}$ and has almost surely Hölder continuous sample paths. The concept of a stationary stochastic process was introduced by Khinchin in [20].

It is well known, following Uhlenbeck and Ornstein's paper [35], that solutions $\left(b_{t}, Z_{t}\right)$ of equation (1.1) exist. The stationary process $Z_{t}$ is unique in distribution and called the 
Ornstein-Uhlenbeck process with parameter $\rho$. We may write $Z_{t}$ as a stochastic spectral integral

$$
Z_{t}=\frac{1}{\sqrt{2 \pi}} \int_{-\infty}^{\infty} \frac{\mathrm{e}^{\mathrm{i} \omega t}}{\rho+\mathrm{i} \omega} \mathrm{d} \tilde{b}_{\omega}
$$

where $\left(\tilde{b}_{\omega}\right)_{\omega \in \mathbb{R}}$ is a given complex Brownian motion on the line defined on $(\Omega, \mathcal{F}, \mathbb{P})$ with $\tilde{b}_{0}=0$. The process $b_{t}$ is given in terms of $\tilde{b}_{\omega}$ by the condition $b_{0}=0$ and the stochastic spectral integral, for $s<t$,

$$
b_{t}-b_{s}=\frac{1}{\sqrt{2 \pi}} \int_{-\infty}^{\infty} \frac{\mathrm{e}^{\mathrm{i} \omega t}-\mathrm{e}^{\mathrm{i} \omega s}}{\mathrm{i} \omega} \mathrm{d} \tilde{b}_{\omega} .
$$

The formulae (1.2) and (1.3) were originally derived by mathematicians developing the theory of linear filters on stationary stochastic processes. Of note are Blanc-Lapierre and Fortet's paper [1] , which discusses the basic properties of filters, and Kolmogorov's paper [21], which discusses spectral representations of solutions to linear constant-coefficient stochastic differential equations: formula (24) of Kolmogorov's paper is a generalized form of (1.2). See Chapter XI of [11] for a detailed description of the spectral theory of scalar-valued stationary stochastic processes.

Adaptedness of the process $Z_{t}$ to the filtration induced by $b_{t}$ follows from the existence of a time domain integral

$$
Z_{t}=\int_{-\infty}^{t} \mathrm{e}^{-\rho(t-u)} \mathrm{d} b_{u}
$$

which expresses $Z_{t}$ as a stochastic integral with respect to $b_{t}$. All stochastic integrals are interpreted in the Itô sense. The autocovariance of the process $Z_{t}$ is

$$
\operatorname{Cov}\left(Z_{s}, Z_{t}\right)=\frac{\mathrm{e}^{-\rho|s-t|}}{2 \rho} .
$$

The Ornstein-Uhlenbeck process is Gaussian, strongly Markovian and stationary with almost surely Hölder continuous sample paths.

For more information, particularly on the physical motivation for studying these processes, we refer the reader to $[\mathbf{1 4}]$ or $[\mathbf{3 3}]$.

In $\S 4$ of this paper we consider a generalization of the Langevin equation to the Banach space valued case. We let $E$ be a separable complex Banach space and consider the stochastic differential equation

$$
\mathrm{d} \boldsymbol{Z}_{t}+\Lambda \boldsymbol{Z}_{t} \mathrm{~d} t=\mathrm{d} \boldsymbol{B}_{t}
$$

for $t \in \mathbb{R}$, where $\Lambda$ is a closed operator from a norm dense domain $\mathcal{D}(\Lambda) \subseteq E$ to $E$.

We seek a pair of processes $\left(\boldsymbol{B}_{t}, \boldsymbol{Z}_{t}\right)$, defined on a probability space $(\Omega, \mathcal{F}, \mathbb{P})$, which are a weak solution to equation (1.6); the concept of a weak solution follows that of Da Prato and Zabczyk, for which see $[6]$, and is defined formally in $\S 4$. The process $\left(\boldsymbol{B}_{t}\right)_{t \in \mathbb{R}}$ is required to be an $E$-valued $Q$-Wiener process; our terminology for Wiener processes follows that used in $[\mathbf{6}]$. The process $\left(\boldsymbol{Z}_{t}\right)_{t \in \mathbb{R}}$ is required to be an $E$-valued, 
centred Gaussian, stationary stochastic process with almost surely Hölder continuous sample paths.

When generalizing results on scalar-valued random variables and stochastic processes to the Banach space valued case, several problems arise concerning how to describe concepts such as expectation, $L^{2}$ boundedness, covariance and stationarity in a wider setting. Section 2 of this paper uses ideas developed by Pisier in [32] to develop the theory of Banach space valued random vectors with bounded variance.

Particular use is made of a norm on spaces of random vectors equal to the 2-absolutely summing norm on an associated space of linear operators; this norm is denoted by $\pi_{2}$. Section 3 of this paper characterizes Gaussian random vectors and $Q$-Wiener processes in a separable Banach space $E$ using the $\pi_{2}$ norm; it is shown that, necessarily, $Q$ factors through $\ell^{2}$ as $A \overline{A^{*}}$, where $A$ is an operator from $\ell^{2}$ to $E$ with 2-summing adjoint. This factorization condition is shown to be sufficient when $E$ is of Gaussian type 2.

Section 3 also considers the theory of stochastic integration in a separable Banach space for continuous deterministic integrands with respect to a $Q$-Wiener process. The following theorem is proved.

Theorem 1.1. For $E$ a separable Banach space, let $\boldsymbol{B}_{t}$ be an $E$-valued $A \overline{A^{*}}$-Wiener process defined on a probability space $(\Omega, \mathcal{F}, \mathbb{P})$. Then for $s<t$, if $\left(T_{u}\right)_{s \leqslant u \leqslant t}$ is a nonrandom family of bounded linear operators on $E$ such that $\left(A^{*} T_{u}^{*}\right)_{s \leqslant u \leqslant t}$ is continuous in the $\pi_{2}$ norm, the stochastic integral

$$
\int_{s}^{t} T_{u} \mathrm{~d} \boldsymbol{B}_{u}
$$

exists as the limit in the $\pi_{2}$ norm of appropriate Riemann sums under refinement of partitions. Furthermore,

$$
\pi_{2}\left(\int_{s}^{t} T_{u} \mathrm{~d} \boldsymbol{B}_{u}\right)^{2} \leqslant \int_{s}^{t} \pi_{2}\left(A^{*} T_{u}^{*}\right)^{2} \mathrm{~d} u .
$$

In cases where $s$ is equal to $-\infty$ and/or $t$ is equal to $\infty$, we define the stochastic integral in a way that is analogous to the improper Riemann integral, that is as the limiting value of the integral as $s$ decreases to $-\infty$ and/or $t$ increases to $\infty$, where this limiting value exists.

Having formalized the framework under which Banach space valued stochastic processes will be discussed, we prove the existence in $\S 4$, under certain boundedness conditions on $\Lambda$, of pairs of processes $\left(\boldsymbol{B}_{t}, \boldsymbol{Z}_{t}\right)$ which solve the Banach space valued Langevin equation in the weak sense.

Theorem 1.2. Assume $\mathrm{i} \Lambda$ and $\mathrm{i} \Lambda^{*}$ generate $C_{0}$ groups of operators on $E$ and the resolvent of $\Lambda$ is uniformly bounded on the imaginary axis. Consider the stochastic spectral integral

$$
\boldsymbol{Z}_{t}=\frac{1}{\sqrt{2 \pi}} \int_{-\infty}^{\infty} \mathrm{e}^{\mathrm{i} \omega t}(\Lambda+\mathrm{i} \omega I)^{-1} \mathrm{~d} \tilde{\boldsymbol{B}}_{\omega}
$$


where $\tilde{\boldsymbol{B}}_{\omega}$ is a given $E$-valued $Q$-Wiener process defined on $(\Omega, \mathcal{F}, \mathbb{P})$. Define $\boldsymbol{B}_{t}$ subject to $\boldsymbol{B}_{0}=0$ and the stochastic spectral integral, for $s<t$,

$$
\boldsymbol{B}_{t}-\boldsymbol{B}_{s}=\frac{1}{\sqrt{2 \pi}} \int_{-\infty}^{\infty} \frac{\mathrm{e}^{\mathrm{i} \omega t}-\mathrm{e}^{\mathrm{i} \omega s}}{\mathrm{i} \omega} \mathrm{d} \tilde{\boldsymbol{B}}_{\omega} .
$$

The processes $\boldsymbol{Z}_{t}$ and $\boldsymbol{B}_{t}$ converge as stochastic integrals and the pair $\left(\boldsymbol{B}_{t}, \boldsymbol{Z}_{t}\right)$ is a weak solution of equation (1.6).

The process $\boldsymbol{Z}_{t}$ is a generalization to the $E$-valued case of the classical OrnsteinUhlenbeck process. Such a generalization has been done previously, notably by Itô in [18]; the difference here is that our solution $\boldsymbol{Z}_{t}$ is represented as a stochastic spectral integral, rather than an integral in the time domain.

Note we do not require that $\boldsymbol{Z}_{t}$ be adapted to the filtration induced by $\boldsymbol{B}_{t}$. We obtain adaptedness in the important case where $(-\Lambda)$ generates a $C_{0}$ semigroup $\left(\mathrm{e}^{-\Lambda t}\right)_{t \geqslant 0}$ of exponential norm decay, however, by demonstrating the existence of a time domain integral

$$
\boldsymbol{Z}_{t}=\int_{-\infty}^{t} \mathrm{e}^{-\Lambda(t-u)} \mathrm{d} \boldsymbol{B}_{u}
$$

which expresses $\boldsymbol{Z}_{t}$ as a stochastic integral with respect to $\boldsymbol{B}_{t}$.

Section 4 also considers some specific examples of $E$-valued Langevin equations and their weak solutions. Each example corresponds to an operator $\Lambda$ for which neither $\Lambda$ nor $(-\Lambda)$ generate $C_{0}$ semigroups of exponential norm decay.

\section{Banach space valued random vectors}

In this section we develop the formalism we need to adequately deal with the theory of Banach space valued random vectors and their covariances. The ideas largely derive from Pisier's paper [32]; further background information may be found in [10], [9] and [31].

Following [32] we use positive sesquilinear forms to define a norm on Banach space valued random vectors equal to the 2-absolutely summing norm on equivalent operators from the dual of that Banach space to a Hilbert space. This norm will enable us to bound various stochastic integrals in $\S 4$.

\subsection{Definitions}

We introduce some notation. For a complex vector space $V, \bar{V}$ denotes $V$ endowed with the conjugate scalar multiplication $(\lambda, v) \mapsto \bar{\lambda} v$. Write $\bar{v}$ for $v \in V$ viewed as an element of $\bar{V}$.

Throughout this paper, all adjoints of linear operators are to be interpreted in the Banach space sense.

Let $E$ and $F$ be Banach spaces, let $B(E)$ and $B(F)$ be the spaces of all bounded linear operators on $E$ and $F$, respectively, and let $B(E, F)$ be the space of all bounded linear operators from $E$ to $F$. We shall consider certain classes of norms on spaces of operators from $E$ to $F$. Let $\mathcal{A}(E, F)$ denote a subspace of $B(E, F)$ equipped with a norm $\alpha$ under 
which $\mathcal{A}(E, F)$ is a Banach space. We say $\mathcal{A}(E, F)$ is a Banach operator ideal, and $\alpha$ is an operator ideal norm, if

(i) for all $\xi^{*} \in E^{*}$ and $\eta \in F$, the rank-one tensor $\xi^{*} \otimes \eta \in \mathcal{A}(E, F)$ and

$$
\alpha\left(\xi^{*} \otimes \eta\right)=\left\|\xi^{*}\right\|_{E^{*}}\|\eta\|_{F}
$$

(ii) for all $u \in \mathcal{A}(E, F), S \in B(E)$ and $T \in B(F)$, the product $T u S \in \mathcal{A}(E, F)$ and

$$
\alpha(T u S) \leqslant\|T\| \alpha(u)\|S\|
$$

Here $\|\cdot\|$ denotes the usual operator norm. The most familiar example of a Banach operator ideal is $B(E, F)$ equipped with $\|\cdot\|$; this is also the largest operator ideal in the sense that, if $\alpha$ is an operator ideal norm on an operator ideal $\mathcal{A}(E, F)$ and $u \in \mathcal{A}(E, F)$, we have

$$
\|u\| \leqslant \alpha(u)
$$

For more information on operator ideals the reader is directed to [10].

We recall from, for example, [10] the definition of a 2-absolutely summing operator. For $E$ and $F$ Banach spaces, the operator $T: E \rightarrow F$ is 2-summing if

$$
\pi_{2}(T)=\sup _{\left\{\xi_{i}\right\}_{i}}\left(\sum_{i}\left\|T \xi_{i}\right\|_{F}^{2}\right)^{1 / 2}<\infty
$$

where the supremum is over all finite subsets $\left\{\xi_{i}\right\}_{i}$ of $E$ satisfying

$$
\sup \left\{\sum_{i}\left|\xi^{*}\left(\xi_{i}\right)\right|^{2}: \xi^{*} \in E^{*},\left\|\xi^{*}\right\|_{E^{*}} \leqslant 1\right\} \leqslant 1 .
$$

The constant $\pi_{2}(T)$ is the 2 -summing norm of $T$; it is an operator ideal norm. We write $\Pi_{2}(E, F)$ for the space of all such $T$; it forms a Banach space with norm $\pi_{2}$ and so is a Banach operator ideal. In the case where $E$ and $F$ are both Hilbert spaces, the space $\Pi_{2}(E, F)$ is the space of Hilbert-Schmidt operators from $E$ to $F$.

Let $E$ be a complex Banach space and $H$ a complex Hilbert space. Let $\mathcal{D}(E, H)$ be a Banach space of operators from $E$ to $H$ equipped with a norm $\delta$ satisfying:

(D1) $\delta$ is an operator ideal norm and $\mathcal{D}(E, H)$ is a Banach operator ideal;

(D2) $\delta(u)=\sup _{P} \delta(P u)$ for all $u \in \mathcal{D}(E, H)$, where $P$ is a finite rank orthogonal projection on $H$; and

(D3) if $u \in \mathcal{D}(E, H)$, then

$$
\|u\| \leqslant \delta(u) \leqslant \pi_{2}(u)
$$

It is straightforward to show that both $B(E, H)$ and $\Pi_{2}(E, H)$ satisfy these properties. 
If $\delta$ is such a norm satisfying (D1)-(D3), we say $\delta$ is 2-convex if

$$
\delta\left(\sum_{k} P_{k} u\right)^{2} \leqslant \sum_{k} \delta\left(P_{k} u\right)^{2}
$$

for all $u \in \mathcal{D}(E, H)$ and each finite set of mutually orthogonal projections $\left(P_{k}\right)$ on $H$. Note that both operator norm $\|\cdot\|$ and 2-summing norm $\pi_{2}$ are 2 -convex; the fact that $\pi_{2}$ is 2 -convex is used repeatedly throughout this paper. For more information on 2-convexity we direct the reader to $\S 2$ of $[\mathbf{3 2}]$.

Type 2 is defined as follows. Let $\left(X_{k}\right)_{k}$ denote a sequence of independent real $N(0,1)$ random variables. A Banach space $E$ is of (Gaussian) type 2 if there exists a finite positive constant $C$ such that, for any finite sequence $\left(\xi_{k}\right)_{k}$ in $E$,

$$
\left(\mathbb{E}\left\|\sum_{k} \xi_{k} X_{k}\right\|_{E}^{2}\right)^{1 / 2} \leqslant C\left(\sum_{k}\left\|\xi_{k}\right\|_{E}^{2}\right)^{1 / 2} .
$$

We denote the infimum of all allowable constants $C$ by $T_{2}(E)$, the type 2 constant of $E$. There are many important examples of type 2 Banach spaces; in particular the Lebesgue spaces $L^{p}$ and the Schatten-von Neumann spaces $c^{p}$ are of type 2 for $2 \leqslant p<\infty$. For more information on the notion of type, and the related notion of cotype, see [10] or [31].

\subsection{A class of weakly measurable random vectors}

In this discussion $(\Omega, \mathcal{F}, \mathbb{P})$ will be a probability space, $E$ will be a complex Banach space, $X$ will be a function from $\Omega$ to $E$, and $K$ will be the collection of all positive sesquilinear forms on $E \times E$ of norm less than or equal to one. We say $X: \Omega \rightarrow E$ is weakly (also known as Pettis) measurable if $\xi^{*} X: \Omega \rightarrow \mathbb{C}$ is Borel measurable for all $\xi^{*} \in E^{*}$. It is clear that the set of all weakly measurable functions from $\Omega$ to $E$ forms a vector space; this is known as the space of cylindrical random vectors.

Let $L^{2}(\Omega)$ be the Hilbert space of complex-valued square-integrable functions on $(\Omega, \mathcal{F}, \mathbb{P})$, quotiented by functions which are zero almost surely. Denote by $\mathcal{L}^{2}(\Omega)$ the corresponding seminormed space of square-integrable functions.

For $X: \Omega \rightarrow E$ weakly measurable and $K$ as above we may, following Pisier in [32], define a seminorm $\delta_{K}$ via the formula

$$
\delta_{K}(X)=\sup _{\varphi \in K}\left(\int_{\Omega} \varphi(X(\omega), X(\omega)) \mathbb{P}(\mathrm{d} \omega)\right)^{1 / 2} .
$$

The only axiom of a seminorm which is unclear is the triangle inequality. However, sesquilinearity and the Cauchy-Schwarz inequality enable us to prove $\left[\delta_{K}\left(X_{1}+X_{2}\right)\right]^{2} \leqslant$ $\left[\delta_{K}\left(X_{1}\right)+\delta_{K}\left(X_{2}\right)\right]^{2}$. Denote the seminormed space of all weakly measurable $X: \Omega \rightarrow E$ with $\delta_{K}(X)<\infty$ by $\mathcal{L}_{w}^{2}\left(\Omega ; E, \delta_{K}\right)$.

We may quotient $\mathcal{L}_{w}^{2}\left(\Omega ; E, \delta_{K}\right)$ by the set of all weakly measurable $X: \Omega \rightarrow E$ with $\delta_{K}(X)=0$ to obtain a normed space, which we denote by $L_{w}^{2}\left(\Omega ; E, \delta_{K}\right)$. This space is not, in general, complete; however, we may embed it isometrically in a Banach space of operators. The following result follows from Proposition 2.1 of [32]. 
Proposition 2.1. There is an isometric embedding

$$
\wedge: L_{w}^{2}\left(\Omega ; E, \delta_{K}\right) \hookrightarrow \Pi_{2}\left(E^{*}, L^{2}(\Omega)\right)
$$

given by the relation

$$
\hat{X}\left(\xi^{*}\right)(\omega)=\xi^{*}(X(\omega)) .
$$

Proof. Referring to Proposition 2.1 of [32], we see that the relation (2.11) defines an isometric embedding $\wedge: \mathcal{L}_{w}^{2}\left(\Omega ; E, \delta_{K}\right) \hookrightarrow \Pi_{2}\left(E^{*}, \mathcal{L}^{2}(\Omega)\right)$ between seminormed spaces.

We may quotient by the appropriate null space to obtain an isometric embedding between normed quotient spaces if, given a random vector $X, \delta_{K}(X)=0$ implies $\pi_{2}(\hat{X})=0$. But this follows, since $\delta_{K}(X)=0$ if and only if $\xi^{*}(X)=0$ almost surely, for all $\xi^{*}$ in $E^{*}$, which occurs if and only if $\pi_{2}(\hat{X})=0$.

We shall henceforth use the notation $\pi_{2}$ to denote both the $\delta_{K}$ norm on $L_{w}^{2}\left(\Omega ; E, \delta_{K}\right)$ and the 2 -summing norm on $\Pi_{2}\left(E^{*}, L^{2}(\Omega)\right)$. The space $L_{w}^{2}\left(\Omega ; E, \delta_{K}\right)$ will now be referred to as $L_{w}^{2}\left(\Omega ; E, \pi_{2}\right)$.

\subsection{The Bochner spaces}

At times we shall also consider the more usual Bochner $L^{p}$ spaces. Following [9] we say $X: \Omega \rightarrow E$ is strongly (also known as Bochner) measurable if it is measurable with respect to the Borel $\sigma$-algebra on $E$ and takes values almost surely in a separable subspace of $E$ (we say it is almost surely separably valued).

If $1 \leqslant p<\infty$ and $X: \Omega \rightarrow E$ is a strongly measurable random vector, then its Bochner $L^{p}$ norm is given by

$$
\|X\|_{p}=\left(\int_{\Omega}\|X(\omega)\|_{E}^{p} \mathbb{P}(\mathrm{d} \omega)\right)^{1 / p} .
$$

Denote by $\mathcal{L}^{p}(\Omega ; E)$ the set of all strongly measurable $X: \Omega \rightarrow E$ with finite Bochner $L^{p}$ norm. This is a seminormed vector space whose null space is the subspace of all strongly measurable $X: \Omega \rightarrow E$ which are zero almost surely. Denote by $L^{p}(\Omega ; E)$ the resulting quotient space; this is a Banach space.

We may define analogous $L^{p}(\Omega ; E)$ in the cases $0<p<1$; the resulting spaces are complete quasinormed spaces.

Note that if $\pi_{2}$ is the weak $L^{2}$ norm introduced in the last subsection and $X$ lies in $L^{2}(\Omega ; E)$, then

$$
\pi_{2}(X) \leqslant\|X\|_{2}
$$

\subsection{Expectation}

Following [9], we say a weakly measurable random vector $X: \Omega \rightarrow E$ is weakly (or Dunford) integrable if $\xi^{*} X \in L^{1}(\Omega)$ for all $\xi^{*} \in E^{*}$. This occurs if and only if

$$
\sup _{\xi^{*} \in E^{*},\left\|\xi^{*}\right\| \leqslant 1} \int_{\Omega}\left|\xi^{*} X(\omega)\right| \mathbb{P}(\mathrm{d} \omega)<\infty .
$$


For a weakly integrable $X: \Omega \rightarrow E$ and $A \in \mathcal{F}$ we see there exists

$$
\int_{A} X(\omega) \mathbb{P}(\mathrm{d} \omega) \in E^{* *}
$$

such that

$$
\left(\int_{A} X(\omega) \mathbb{P}(\mathrm{d} \omega)\right)\left(\xi^{*}\right)=\int_{A} \xi^{*} X(\omega) \mathbb{P}(\mathrm{d} \omega)
$$

for all $\xi^{*} \in E^{*}$. When $A=\Omega$ we refer to the integral as the weak expectation $\mathbb{E} X$ of $X$ with respect to $\mathbb{P}$.

We note that if $X \in L_{w}^{2}\left(\Omega ; E, \pi_{2}\right)$, then, by the Cauchy-Schwarz inequality, the above condition holds; thus the weak expectation exists and is finite as an element of $E^{* *}$.

It may be of course that

$$
\int_{A} X(\omega) \mathbb{P}(\mathrm{d} \omega) \in E
$$

for all $A \in \mathcal{F}$. In this case, following [9], we say $X$ is Pettis integrable; in particular $\mathbb{E} X \in E$.

Finally, if $X$ lies in the Bochner space $L^{1}(\Omega ; E)$, we say, following [9], that it is Bochner (or strongly) integrable. Bochner integrability implies Pettis integrability.

The following result gives a sufficient condition for a Cauchy sequence in $L_{w}^{2}\left(\Omega ; E, \pi_{2}\right)$ to converge in that space; recall that, a priori, we only know that the limit exists as an operator in $\Pi_{2}\left(E^{*}, L^{2}(\Omega)\right)$.

Proposition 2.2. If $\left(X_{n}\right)_{n \geqslant 1}$ is a sequence in $L^{2}(\Omega ; E)$ which is Cauchy in the $\pi_{2}$ norm, then the sequence converges in $L_{w}^{2}\left(\Omega ; E, \pi_{2}\right)$.

Proof. Viewing $\left(X_{n}\right)_{n \geqslant 1}$ as a sequence in the Banach operator ideal $\Pi_{2}\left(E^{*}, L^{2}(\Omega)\right)$ we see that, as it is a Cauchy sequence in the $\pi_{2}$ norm, it must converge in $\Pi_{2}\left(E^{*}, L^{2}(\Omega)\right)$ to a limit operator $\hat{X}$. By Theorem VIII.1.5 of $[\mathbf{9}]$, as $\left(X_{n}\right)_{n \geqslant 1}$ is a sequence in $L^{2}(\Omega ; E)$, the operator $\hat{X}$ has compact adjoint $\hat{X}^{*}$ taking values in a separable subspace of $E$. By Theorem III.2.2 of [9] the compact operator $\hat{X}^{*}: L^{2}(\Omega) \rightarrow E$ is representable, which means there exists a weakly measurable $X: \Omega \rightarrow E$ satisfying

$$
\hat{X}^{*}(f)=\int_{\Omega} X(\omega) f(\omega) \mathbb{P}(\mathrm{d} \omega)
$$

for each $f$ in $L^{2}(\Omega)$. But this $X$ is transparently the limit in $L_{w}^{2}\left(\Omega ; E, \pi_{2}\right)$ of the sequence $\left(X_{n}\right)_{n \geqslant 1}$.

\subsection{Covariance}

Let $E_{1}$ and $E_{2}$ be Banach spaces and let $X_{1}$ and $X_{2}$ be elements of $L_{w}^{2}\left(\Omega ; E_{1}, \pi_{2}\right)$ and $L_{w}^{2}\left(\Omega ; E_{2}, \pi_{2}\right)$, respectively. Assume $\mathbb{E} X_{1}=\mathbb{E} X_{2}=0$, where expectation is defined in the weak sense; we say $X_{1}$ and $X_{2}$ are centred.

Define the covariance of $X_{1}$ and $X_{2}, \operatorname{Cov}\left(X_{1}, X_{2}\right)$, to be the sesquilinear form on $E_{1}^{*} \times E_{2}^{*}$ given by

$$
\operatorname{Cov}\left(X_{1}, X_{2}\right)\left(\xi_{1}^{*}, \xi_{2}^{*}\right)=\mathbb{E} \xi_{1}^{*}\left(X_{1}\right) \overline{\xi_{2}^{*}\left(X_{2}\right)}
$$


This definition of covariance is used in [26] and [28]. We note by the Cauchy-Schwarz inequality that $\operatorname{Cov}\left(X_{1}, X_{2}\right)$ is bounded as a sesquilinear form.

Frequently we view $\operatorname{Cov}\left(X_{1}, X_{2}\right)$ as a linear operator from $\overline{E_{2}^{*}}$ to $E_{1}^{* *}$; in fact it is the operator $\hat{X}_{1}^{*} \hat{X}_{2}$, where, as before, $\hat{X}_{1}: E_{1}^{*} \rightarrow L^{2}(\Omega)$ and $\hat{X}_{2}: E_{2}^{*} \rightarrow L^{2}(\Omega)$ are the operators associated to $X_{1}$ and $X_{2}$. For more information on this subject the reader is directed to Chapter 2 of [15].

If $E$ is a Banach space and $X$ is a centred element of $L_{w}^{2}\left(\Omega ; E, \pi_{2}\right)$, we define the variance of $X, \operatorname{Var}(X)$, to be $\operatorname{Cov}(X, X)$.

Remark 2.3. Throughout the rest of this paper we shall, to avoid measure-theoretic complications (which are expanded on in $[\mathbf{1 5}]$ and $[\mathbf{2 9}]$ ), assume all the Banach spaces under consideration are separable. Thus, in particular, strong (Bochner) and weak (Pettis) notions of measurability coincide. All the random vectors we consider in the rest of the paper will be cylindrical; that is to say they will be measurable with respect to the cylindrical $\sigma$-algebra on the Banach space, which, as the Banach space is separable, coincides with the Borel $\sigma$-algebra.

\section{Gaussian random vectors and Wiener processes}

This section contains essential preliminary material for $\S 4$. Background information on the topics covered here may be found in $[\mathbf{6}],[\mathbf{7}],[\mathbf{2 4}]$ or $[\mathbf{2 6}]$. We study Gaussian random vectors, Wiener processes and stochastic integrals (for continuous deterministic integrands) with values in a separable complex Banach space $E$. We observe that, for all $E$-valued $Q$-Wiener processes on a probability space $(\Omega, \mathcal{F}, \mathbb{P}), Q$ factors through $\ell^{2}$ with 2 -summing factors.

For more general integrands, the theory of stochastic integration in a Banach space becomes rather more involved; see, for example, [2] and [3], in which the theory is developed for Banach spaces of martingale type 2 .

\subsection{Gaussian random vectors}

We will consider centred Gaussian random vectors taking values in a separable complex Banach space $E$.

Recall that a complex random variable $X$ is said to be complex centred Gaussian with variance $\sigma^{2}$ (we say $X$ is complex $N\left(0, \sigma^{2}\right)$ ) if

$$
X=\frac{1}{\sqrt{2}}\left(X_{\mathcal{R}}+\mathrm{i} X_{\mathcal{I}}\right)
$$

where $X_{\mathcal{R}}$ and $X_{\mathcal{I}}$ are independent real $N\left(0, \sigma^{2}\right)$ random variables. It is straightforward to verify that if $\left(X_{k}\right)_{k}$ is a finite sequence of independent complex centred Gaussian random vectors on some probability space and $\left(z_{k}\right)_{k}$ is a finite sequence in $\mathbb{C}$, then $\sum_{k} z_{k} X_{k}$ is a complex centred Gaussian random vector.

Let $X$ be an $E$-valued cylindrical random vector defined on a probability space $(\Omega, \mathcal{F}, \mathbb{P})$. Following $[\mathbf{2 6}]$ we say $X$ is centred Gaussian if, for every $\xi^{*}$ in $E^{*}, \xi^{*}(X)$ is a complex centred Gaussian random variable. 
The following result is a combination of the Itô-Nisio Theorem [19], a result on exponential integrability of Gaussian random vectors, due independently to Fernique [12] and Landau and Shepp [25] and the Karhunen-Loève representation of Gaussian measures on separable Banach spaces.

\section{Proposition 3.1.}

(a) Let $\boldsymbol{X}=\left(X_{k}\right)_{k \in \mathbb{Z}}$ be a sequence of independent $N\left(0, \sigma^{2}\right)$ complex random variables on a probability space $(\Omega, \mathcal{F}, \mathbb{P})$ and let $\left(\xi_{k}\right)_{k \in \mathbb{Z}}$ be a sequence in a separable complex Banach space $E$. The following are equivalent.

(i) $\sum_{k} \xi_{k} X_{k}$ converges almost surely in $E$.

(ii) $\sum_{k} \xi_{k} X_{k}$ converges in $L^{p}(\Omega ; E)$ for some (and hence for all) $0<p<\infty$.

(iii) $\sum_{k} \xi_{k} X_{k}$ converges in probability.

(b) If $\left(Y_{k}\right)_{k \geqslant 1}$ is any sequence of cylindrical centred Gaussian random vectors, defined on $(\Omega, \mathcal{F}, \mathbb{P})$ with values in $E$, which is Cauchy in the $\pi_{2}$ norm, then the sequence converges in $L_{w}^{2}\left(\Omega ; E, \pi_{2}\right)$ to a cylindrical centred Gaussian random vector.

(c) All cylindrical centred Gaussian random vectors with values in $E$ are equal in distribution to some random vector of the form $\sum_{k} \xi_{k} X_{k}$, satisfying the equivalent conditions (i), (ii) and (iii) of part (a).

Proof. (a) This is the Itô-Nisio Theorem; see the original paper [19], or pp. 29-36 of $[\mathbf{2 4}]$, for details.

(b) We will use the result, due to Fernique [12] and Landau and Shepp [25], that for $Z$ any centred Gaussian random vector with values in $E$, there exists $\alpha>0$ such that

$$
\mathbb{E} \exp \left\{\alpha\|Z\|_{E}^{2}\right\}<\infty
$$

in particular $Z$ lies in $L^{2}(\Omega ; E)$.

It follows that $\left(Y_{k}\right)_{k \geqslant 1}$ is a sequence in $L^{2}(\Omega ; E)$. Proposition 2.2 shows it converges in $L_{w}^{2}\left(\Omega ; E, \pi_{2}\right)$ to a limit $Y$. We shall show that $Y$ is a centred Gaussian random vector.

The definition of the $\pi_{2}$ norm shows that, for each $\xi^{*}$ in $E^{*}$, the sequence $\left(\xi^{*}\left(Y_{k}\right)\right)_{k \geqslant 1}$ converges in $L^{2}(\Omega)$ to $\xi^{*}(Y)$. As each $\xi^{*}\left(Y_{k}\right)$ is a complex centred Gaussian random variable, it follows that $\xi^{*}(Y)$ is a complex centred Gaussian random variable.

Since this holds for all $\xi^{*}$ in $E^{*}$, we deduce that $Y$ is a centred Gaussian random vector.

(c) The Karhunen-Loève representation of Gaussian measures, which is Proposition 2.6.1 of [24] and Proposition 3.6 of [26], now shows that all cylindrical centred Gaussian random vectors with values in $E$ are equal in distribution to some random vector of the form $\sum_{k} \xi_{k} X_{k}$, satisfying conditions (i), (ii) and (iii) of part (a).

Proposition 3.1 has the following corollary. 
Corollary 3.2. Given a Gaussian random vector $\sum_{k} \xi_{k} X_{k}$ as in Proposition 3.1, define a map $A$ from the space of those complex sequences $\left(x_{k}\right)_{k \in \mathbb{Z}}$, such that all but a finite number of the $x_{k}$ are zero, to $E$ by

$$
\left(x_{k}\right)_{k \in \mathbb{Z}} \mapsto \sum_{k} \xi_{k} x_{k} ;
$$

this extends to a bounded operator $A: \ell^{2} \rightarrow E$ with bounded adjoint $A^{*} \in \Pi_{2}\left(E^{*}, \ell^{2}\right)$.

Write $A \boldsymbol{X}$ for $\sum_{k} \xi_{k} X_{k}$; this converges to an almost surely E-valued centred Gaussian random vector satisfying $\pi_{2}(A \boldsymbol{X})=\sigma \pi_{2}\left(A^{*}\right)$ and $\operatorname{Var}(A \boldsymbol{X})=\sigma^{2} A \overline{A^{*}}$.

Proof. Writing $A \boldsymbol{X}$ for $\sum_{k} \xi_{k} X_{k}$, we know from Proposition 3.1 that $A \boldsymbol{X}$ defines an almost surely $E$-valued element of $L^{2}(\Omega ; E)$. As the $\pi_{2}$ norm is weaker than Bochner $L^{2}$ norm, it follows that $A \boldsymbol{X}$ lies in $L_{w}^{2}\left(\Omega ; E, \pi_{2}\right)$; we now apply Proposition 2.1 to observe that the map $\left(\xi^{*} \mapsto \xi^{*}(A \boldsymbol{X})\right)$ lies in $\Pi_{2}\left(E^{*}, L^{2}(\Omega)\right)$. But, for a finite sequence $\left(\xi_{j}^{*}\right)_{j}$ in $E^{*}$,

$$
\begin{aligned}
\sum_{j}\left\|\xi_{j}^{*}(A \boldsymbol{X})\right\|_{L^{2}(\Omega)}^{2} & =\sum_{j} \mathbb{E}\left|\sum_{k} \xi_{j}^{*}\left(\xi_{k}\right) X_{k}\right|^{2} \\
& =\sigma^{2} \sum_{j, k}\left|\xi_{j}^{*}\left(\xi_{k}\right)\right|^{2} \\
& =\sigma^{2} \sum_{j}\left\|A^{*}\left(\xi_{j}^{*}\right)\right\|_{\ell^{2}}^{2}
\end{aligned}
$$

and so $A^{*} \in \Pi_{2}\left(E^{*}, \ell^{2}\right)$; furthermore, $\sigma \pi_{2}\left(A^{*}\right)=\pi_{2}(A \boldsymbol{X})$. As $A^{*}$ is bounded it follows that its adjoint $A^{* *}: \ell^{2} \rightarrow E^{* *}$ is bounded. We see, however, that $A^{* *}$ is equal to $\iota A$, where $\iota$ denotes the isometric embedding of $E$ into $E^{* *}$. This implies that $A$ is bounded from $\ell^{2}$ to $E$. Finally,

$$
\operatorname{Var}(A \boldsymbol{X})\left(\xi_{1}^{*}, \xi_{2}^{*}\right)=\sigma^{2}\left\langle A^{*}\left(\xi_{1}^{*}\right), A^{*}\left(\xi_{2}^{*}\right)\right\rangle_{\ell^{2}},
$$

yielding $\operatorname{Var}(A \boldsymbol{X})=\sigma^{2} A \overline{A^{*}}$ as required.

For definiteness we ensure $A \boldsymbol{X}$ is always $E$ valued by defining $A \boldsymbol{X}$ to be $\sum_{k} \xi_{k} X_{k}$ at sample points where this sum converges, and zero on the null set where it diverges.

If $E$ is of type 2, we may deduce more.

Corollary 3.3. Under the additional hypothesis that $E$ is of type 2, the sum $A \boldsymbol{X}$ converges to a centred Gaussian random vector if and only if $A^{*} \in \Pi_{2}\left(E^{*}, \ell^{2}\right)$.

Proof. If $A \boldsymbol{X}$ is centred Gaussian we know from Corollary 3.2 that $A^{*} \in \Pi_{2}\left(E^{*}, \ell^{2}\right)$. Conversely, let us assume $A^{*} \in \Pi_{2}\left(E^{*}, \ell^{2}\right)$. Then by (0.6) on p. 67 of [32], which is based on work in $[\mathbf{1 3}]$, we have

$$
\left(\mathbb{E}\left\|\sum_{k} \xi_{k} X_{k}\right\|_{E}^{2}\right)^{1 / 2} \leqslant \sigma T_{2}(E) \pi_{2}\left(A^{*}\right)<\infty .
$$

We deduce that $A \boldsymbol{X}$ lies in $L^{2}(\Omega ; E)$ and so, by Proposition 3.1, is centred Gaussian. 
Corollary 3.2 enables us to determine, for $E$ a separable complex Banach space, a factorization property for operators $Q: \overline{E^{*}} \rightarrow E^{* *}$ which are variances of centred Gaussian random vectors in $E$. In the case where $E$ is of type 2, Corollary 3.3 enables us to characterize such operators $Q$ precisely.

Corollary 3.4. Let $E$ be a separable complex Banach space.

(a) Let $Q$ be the variance of some centred Gaussian random vector in $E$, defined on some probability space $(\Omega, \mathcal{F}, \mathbb{P})$. Then $Q$ factors as $A \overline{A^{*}}$, where the operator $A: \ell^{2} \rightarrow E$ has 2-summing adjoint.

(b) If, furthermore, $E$ is of type 2 , then conversely any operator $A \overline{A^{*}}$, where $A: \ell^{2} \rightarrow E$ has 2-summing adjoint, is the variance of some centred Gaussian random vector in E.

Proof. (a) Let $Q=\operatorname{Var} \boldsymbol{Z}$, where $\boldsymbol{Z}$ is a centred Gaussian random vector in $E$, defined on $(\Omega, \mathcal{F}, \mathbb{P})$. By Proposition 3.1 (c) and Corollary 3.2, $\boldsymbol{Z}$ lies in $L^{2}(\Omega ; E)$ and is equal in distribution to $\boldsymbol{A} \boldsymbol{X}$, where $\boldsymbol{X}$ is a sequence of independent $N(0,1)$ complex random variables and $A: \ell^{2} \rightarrow E$ is an operator with 2-summing adjoint. Corollary 3.2 now shows $\operatorname{Var}(A \boldsymbol{X})=A \overline{A^{*}}$. Thus $Q=A \overline{A^{*}}$ as required.

(b) Taking $A$ as given, by Corollary 3.3 the random vector $A \boldsymbol{X}$, for $\boldsymbol{X}$ a sequence of independent $N(0,1)$ complex random variables, is a centred Gaussian random vector with variance $A \overline{A^{*}}$.

In [36], van Neerven discusses, for $E$ a separable Banach space, which necessary and sufficient conditions are needed for an operator $\overline{E^{*}} \rightarrow E^{* *}$ to be the variance of a cylindrical Gaussian measure on $E$. We see that Corollary 3.4 provides such conditions in the case where $E$ is of type 2. Similar results for type 2 spaces were obtained by Linde, Tarieladze and Čobanjan in [27].

\subsection{Wiener processes}

We now consider $E$-valued Wiener processes where, as usual, $E$ is a separable complex Banach space. Let $Q$ be the variance of some cylindrical centred Gaussian random vector in $E$.

Following [6] or Chapter 5 of $[\mathbf{7}]$ we say an $E$-valued stochastic process $\left(\boldsymbol{B}_{t}\right)_{t \in \mathbb{R}}$, defined on a probability space $(\Omega, \mathcal{F}, \mathbb{P})$, is a $Q$-Wiener process if

(i) for each $t, \boldsymbol{B}_{t}$ is measurable with respect to the cylindrical $\sigma$-algebra on $E$;

(ii) the process $\boldsymbol{B}_{t}$ has almost surely continuous sample paths and $\boldsymbol{B}_{0}=0$;

(iii) the process $\boldsymbol{B}_{t}$ has independent increments; and

(iv) for each $s<t, \boldsymbol{B}_{t}-\boldsymbol{B}_{s}$ is a cylindrical centred Gaussian random vector satisfying

$$
\operatorname{Var}\left(\boldsymbol{B}_{t}-\boldsymbol{B}_{s}\right)=Q(t-s) .
$$


Condition (i) ensures that, for all $\xi^{*} \in E^{*}$, the process $\xi^{*}\left(\boldsymbol{B}_{t}\right)$ is adapted to the filtration induced on $(\Omega, \mathcal{F}, \mathbb{P})$ by the process $\boldsymbol{B}_{t}$.

Proposition 3.1 and Corollaries 3.2 and 3.4 enable us to deduce various properties of a $Q$-Wiener process.

Proposition 3.5. Let $E$ be a separable Banach space and let $\boldsymbol{B}_{t}$ be an $E$-valued $Q$-Wiener process defined on a probability space $(\Omega, \mathcal{F}, \mathbb{P})$. Let $Q$ factor as $A \overline{A^{*}}$, where $A: \ell^{2} \rightarrow E$ is some operator with 2-summing adjoint given by Corollary 3.4. For $s<t$,

(i) $\pi_{2}\left(\boldsymbol{B}_{t}-\boldsymbol{B}_{s}\right)=\pi_{2}\left(A^{*}\right)(t-s)^{1 / 2}$; and

(ii) for $T$ any bounded linear operator on $E$,

$$
\operatorname{Var}\left(T\left(\boldsymbol{B}_{t}-\boldsymbol{B}_{s}\right)\right)=T Q \overline{T^{*}}(t-s) .
$$

Proof. We observe $\boldsymbol{B}_{t}-\boldsymbol{B}_{s}$ is a cylindrical centred Gaussian random vector satisfying $\operatorname{Var}\left(\boldsymbol{B}_{t}-\boldsymbol{B}_{s}\right)=Q(t-s)$. Proposition $3.1(\mathrm{c})$ and Corollary 3.2 show that $\boldsymbol{B}_{t}-\boldsymbol{B}_{s}$ is equal in distribution to a random vector of the form $A \boldsymbol{X}^{(s, t)}$, where, for each $s$ and $t$, $\boldsymbol{X}^{(s, t)}=\left(X_{k}^{(s, t)}\right)_{k \in \mathbb{Z}}$ is a sequence of independent $N(0, t-s)$ complex random variables. Corollary 3.2 now gives the required results immediately.

Remark 3.6. Given any such $Q$ we may explicitly construct a $Q$-Wiener process. Assume from Corollary 3.4 that $Q$ factors as $A \overline{A^{*}}$. Let $\boldsymbol{b}_{t}=\left(b_{t}^{(k)}\right)_{k \in \mathbb{Z}}$ be an independent sequence of complex Brownian motions on the line, with $b_{0}^{(k)}=0$ for each $k$, defined on the canonical probability space of continuous paths $\mathbb{R} \rightarrow \mathbb{C}$ equipped with Wiener measure. The Ciesielski representation of Brownian motion, for which see [5] or pp. 10-13 of [33], allows us to write

$$
b_{t}^{(k)}(\omega)=\lim _{n \rightarrow \infty} \sum_{r=0}^{n} \sum_{s \in \mathbb{Z}} f_{r s}(t) Z_{r s}^{(k)}(\omega)
$$

for each $k$, where the $f_{r s}$ are tent-shaped functions of maximum value $2^{-(r+1) / 2}$ supported on $\left[(2 s-2) 2^{-r},(2 s) 2^{-r}\right]$, the $Z_{r s}^{(k)}$ are independent complex $N(0,1)$ random variables and the limit is uniform on compact subsets of $\mathbb{R}$ almost surely. Set $\boldsymbol{Z}_{r s}=\left(Z_{r s}^{(k)}\right)_{k \in \mathbb{Z}}$. By applying the same arguments used to prove the existence of the Ciesielski representation on pp. 10-13 of [33] we may define

$$
A \boldsymbol{b}_{t}(\omega)=\lim _{n \rightarrow \infty} \sum_{r=0}^{n} \sum_{s \in \mathbb{Z}} f_{r s}(t) A \boldsymbol{Z}_{r s}(\omega),
$$

which may be shown to be a $Q$-Wiener process.

\subsection{Stochastic integration}

We wish to develop the theory of stochastic integration of a continuous deterministic family of operators with respect to a $Q$-Wiener process. For $s<t$, let $\left(T_{u}\right)_{s \leqslant u \leqslant t}$ be a 
non-random family of bounded linear operators on $E$ and let $\boldsymbol{B}_{t}$ be a $Q$-Wiener process in $E$. Consider a sequence $\left(\mathcal{P}_{n}\right)_{n \geqslant 1}$ of refining partitions of $[s, t]$. Thus, if

$$
\mathcal{P}_{n}=\left\{s=u_{0}^{(n)}<u_{1}^{(n)}<\cdots<u_{r(n)-1}^{(n)}<u_{r(n)}^{(n)}=t\right\}
$$

for each $n$, we assume that $\mathcal{P}_{n} \subseteq \mathcal{P}_{n+1}$ for all $n$ and $\sup _{j}\left(u_{j+1}^{(n)}-u_{j}^{(n)}\right) \downarrow 0$ as $n$ tends to infinity. We say the stochastic integral

$$
\int_{s}^{t} T_{u} \mathrm{~d} \boldsymbol{B}_{u}
$$

exists as a limit in $L_{w}^{2}\left(\Omega ; E, \pi_{2}\right)$ if the sequence of Riemann sums

$$
\sum_{j=0}^{r(n)-1} T_{u_{j}^{(n)}}\left(\boldsymbol{B}_{u_{j+1}^{(n)}}-\boldsymbol{B}_{u_{j}^{(n)}}\right)
$$

converges to a limit in $L_{w}^{2}\left(\Omega ; E, \pi_{2}\right)$ as $n$ tends to infinity, this limit being independent of the choice of partitions $\left(\mathcal{P}_{n}\right)_{n \geqslant 1}$.

We have the following theorem.

Theorem 3.7. For $E$ a separable Banach space, let $\boldsymbol{B}_{t}$ be an $E$-valued $A \overline{A^{*}}$-Wiener process defined on a probability space $(\Omega, \mathcal{F}, \mathbb{P})$. Then for $s<t$, if $\left(T_{u}\right)_{s \leqslant u \leqslant t}$ is a nonrandom family of bounded linear operators on $E$ such that $\left(A^{*} T_{u}^{*}\right)_{s \leqslant u \leqslant t}$ is continuous in the $\pi_{2}$ norm, the stochastic integral

$$
\int_{s}^{t} T_{u} \mathrm{~d} \boldsymbol{B}_{u}
$$

exists as a limit in $L_{w}^{2}\left(\Omega ; E, \pi_{2}\right)$, defining a centred Gaussian element of $L^{2}(\Omega ; E)$. Furthermore,

$$
\pi_{2}\left(\int_{s}^{t} T_{u} \mathrm{~d} \boldsymbol{B}_{u}\right)^{2} \leqslant \int_{s}^{t} \pi_{2}\left(A^{*} T_{u}^{*}\right)^{2} \mathrm{~d} u
$$

Proof. For a partition $\mathcal{P}=\left\{s=u_{0}<u_{1}<\cdots<u_{r}=t\right\}$ of the interval $[s, t]$, put

$$
\begin{aligned}
I(\mathcal{P}) & =\sum_{j=0}^{r-1} T_{u_{j}}\left(\boldsymbol{B}_{u_{j+1}}-\boldsymbol{B}_{u_{j}}\right) \\
& =\sum_{j=0}^{r-1} I\left(u_{j}, u_{j+1}\right),
\end{aligned}
$$

say. Consider a sequence $\left(\mathcal{P}_{n}\right)_{n \geqslant 1}$ of refining partitions of $[s, t]$; with the notation of (3.11), write

$$
\mathcal{M}_{n}=\sup _{j}\left(u_{j+1}^{(n)}-u_{j}^{(n)}\right),
$$

so $\mathcal{M}_{n} \downarrow 0$ as $n$ tends to infinity. Fix $n$ and choose $m>n$. 
To simplify our notation write $u_{j}$ for $u_{j}^{(n)}$, the elements of $\mathcal{P}_{n}$, and write $r$ for $r(n)$, the number of elements of $\mathcal{P}_{n}$ plus one. As $\mathcal{P}_{m}$ is a refinement of $\mathcal{P}_{n}$ we may write it as

$$
\mathcal{P}_{m}=\left\{s=u_{0}=u_{0}^{0}<u_{0}^{1}<\cdots<u_{0}^{k(0)}=u_{1}=u_{1}^{0}<\cdots<u_{r-1}^{k(r-1)}=u_{r}=t\right\} .
$$

Thus

$$
\begin{aligned}
I\left(\mathcal{P}_{m}\right)-I\left(\mathcal{P}_{n}\right) & =\sum_{j=0}^{r-1}\left[\sum_{l=0}^{k(j)-1} I\left(u_{j}^{l}, u_{j}^{l+1}\right)-I\left(u_{j}^{0}, u_{j}^{k(j)}\right)\right] \\
& =\sum_{j=0}^{r-1}\left[\sum_{l=0}^{k(j)-1} T_{u_{j}^{l}}\left(\boldsymbol{B}_{u_{j}^{l+1}}-\boldsymbol{B}_{u_{j}^{l}}\right)-T_{u_{j}^{0}}\left(\boldsymbol{B}_{u_{j}^{k(j)}}-\boldsymbol{B}_{u_{j}^{0}}\right)\right] \\
& =\sum_{j=0}^{r-1}\left[\sum_{l=0}^{k(j)-1} T_{u_{j}^{l}}\left(\boldsymbol{B}_{u_{j}^{l+1}}-\boldsymbol{B}_{u_{j}^{l}}\right)-\sum_{l=0}^{k(j)-1} T_{u_{j}^{0}}\left(\boldsymbol{B}_{u_{j}^{l+1}}-\boldsymbol{B}_{u_{j}^{l}}\right)\right] \\
& =\sum_{j=0}^{r-1} \sum_{l=0}^{k(j)-1}\left(T_{u_{j}^{l}}-T_{u_{j}^{0}}\right)\left(\boldsymbol{B}_{u_{j}^{l+1}}-\boldsymbol{B}_{u_{j}^{l}}\right) .
\end{aligned}
$$

Now the norm $\pi_{2}$ is 2-convex; see $\S 2$ of this paper or $\S 2$ of [32] for details of this concept. This implies

$$
\begin{aligned}
\left\|I\left(\mathcal{P}_{m}\right)-I\left(\mathcal{P}_{n}\right)\right\|_{\pi_{2}}^{2} & \leqslant \sum_{j=0}^{r-1} \sum_{l=0}^{k(j)-1} \pi_{2}\left(A^{*}\left(T_{u_{j}^{l}}^{*}-T_{u_{j}^{0}}^{*}\right)\right)^{2}\left(u_{j}^{l+1}-u_{j}^{l}\right) \\
& \leqslant \sup _{[\alpha, \beta] \subseteq[s, t]: \beta-\alpha \leqslant \mathcal{M}_{n}} \pi_{2}\left(A^{*}\left(T_{\beta}^{*}-T_{\alpha}^{*}\right)\right)^{2}(t-s),
\end{aligned}
$$

and so, for all $m>n$,

$$
\left\|I\left(\mathcal{P}_{m}\right)-I\left(\mathcal{P}_{n}\right)\right\|_{\pi_{2}} \leqslant \sup _{[\alpha, \beta] \subseteq[s, t]: \beta-\alpha \leqslant \mathcal{M}_{n}} \pi_{2}\left(A^{*}\left(T_{\beta}^{*}-T_{\alpha}^{*}\right)\right)(t-s)^{1 / 2} .
$$

We have assumed $u \mapsto A^{*} T_{u}^{*}$ is continuous in the $\pi_{2}$ norm; as $[s, t]$ is compact it follows that $u \mapsto A^{*} T_{u}^{*}$ is uniformly continuous. Thus, fixing $\varepsilon>0$, we may choose $N(\varepsilon)$ such that

$$
\sup _{[\alpha, \beta] \subseteq[s, t]: \beta-\alpha \leqslant \mathcal{M}_{n}} \pi_{2}\left(A^{*}\left(T_{\beta}^{*}-T_{\alpha}^{*}\right)\right)<\varepsilon
$$

whenever $n \geqslant N(\varepsilon)$. Hence, for all $m>n \geqslant N(\varepsilon)$,

$$
\left\|I\left(\mathcal{P}_{m}\right)-I\left(\mathcal{P}_{n}\right)\right\|_{\pi_{2}}<\varepsilon(t-s)^{1 / 2},
$$

which shows $\left(I\left(\mathcal{P}_{n}\right)\right)_{n \geqslant 1}$ is a Cauchy sequence of centred Gaussian random vectors in $L_{w}^{2}\left(\Omega ; E, \pi_{2}\right)$. By Proposition $3.1(\mathrm{~b})$ it therefore converges in $L_{w}^{2}\left(\Omega ; E, \pi_{2}\right)$ to a centred Gaussian random vector. Proposition 3.1 (c) shows that this vector lies in $L^{2}(\Omega ; E)$.

If $\left(\mathcal{P}_{n}\right)_{n \geqslant 1}$ and $\left(\mathcal{P}_{n}^{\prime}\right)_{n \geqslant 1}$ are any two such sequences of partitions of $[s, t]$, it is clear that the sequence of partitions $\left(\mathcal{P}_{n} \cup \mathcal{P}_{n}^{\prime}\right)_{n} \geqslant 1$ also yields a convergent sequence of Riemann 
sums; furthermore, the limits induced by $\left(\mathcal{P}_{n}\right)_{n \geqslant 1},\left(\mathcal{P}_{n}^{\prime}\right)_{n \geqslant 1}$ and $\left(\mathcal{P}_{n} \cup \mathcal{P}_{n}^{\prime}\right)_{n \geqslant 1}$ must coincide. Thus the limit of the sequence $\left(I\left(\mathcal{P}_{n}\right)\right)_{n \geqslant 1}$ in $L_{w}^{2}\left(\Omega ; E, \pi_{2}\right)$ is independent of the choice of refining partitions. We deduce that the stochastic integral exists as a limit in $L_{w}^{2}\left(\Omega ; E, \pi_{2}\right)$.

Finally, by the 2-convexity of the norm $\pi_{2}$, if $s=u_{0}<u_{1}<\cdots<u_{r}=t$ is any partition of $[s, t]$, we have

$$
\pi_{2}\left(\sum_{j=0}^{r-1} T_{u_{j}}\left(\boldsymbol{B}_{u_{j+1}}-\boldsymbol{B}_{u_{j}}\right)\right)^{2} \leqslant \sum_{j=0}^{r-1} \pi_{2}\left(A^{*} T_{u_{j}}^{*}\right)^{2}\left(u_{j+1}-u_{j}\right) .
$$

Passing to the limit gives the required result.

In cases where the lower limit is equal to $-\infty$ and/or the upper limit is equal to $\infty$, we define the corresponding stochastic integral to be the limit in $L_{w}^{2}\left(\Omega ; E, \pi_{2}\right)$, where this exists, of the stochastic integral over $[s, t]$ as $s$ decreases to $-\infty$ and/or $t$ increases to $\infty$.

Theorem 3.7 will be used in the next section to prove the existence of a solution to a Banach space valued Langevin equation.

\section{Ornstein-Uhlenbeck processes}

We now study a Langevin equation for stochastic processes with values in a separable complex Banach space $E$. Background information on diffusion processes may be found in [33] or [34]. Information on semigroups of operators on Banach spaces may be found in $[\mathbf{8}]$ or $[\mathbf{1 7}]$. General information on infinite-dimensional stochastic differential equations may be found in $[\mathbf{6}]$ or Chapter 5 of [7].

Itô studied infinite-dimensional Ornstein-Uhlenbeck processes in the time domain (see [18]). More recently, Kolsrud studied Ornstein-Uhlenbeck processes from the standpoint of Gaussian random fields in [22], Millet and Smoleński investigated the existence and continuity of infinite-dimensional Ornstein-Uhlenbeck processes in [30], van Neerven studied Ornstein-Uhlenbeck semigroups in [36] and Brzeźniak and van Neerven developed stochastic convolution and applied it to infinite-dimensional Ornstein-Uhlenbeck processes in $[4]$.

\subsection{The Langevin equation}

Let $E$ be a separable complex Banach space and $(\Omega, \mathcal{F}, \mathbb{P})$ be a probability space which we assume rich enough to support all the random vectors under consideration. Consider the $E$-valued stochastic differential equation

$$
\mathrm{d} \boldsymbol{Z}_{t}+\Lambda \boldsymbol{Z}_{t} \mathrm{~d} t=\mathrm{d} \boldsymbol{B}_{t}
$$

for $t \in \mathbb{R}$, where

(L1) the operator $\Lambda$ is a closed operator from a norm dense domain $\mathcal{D}(\Lambda) \subseteq E$ to $E$.

We seek a pair of processes $\left(\boldsymbol{B}_{t}, \boldsymbol{Z}_{t}\right)$, each defined on $(\Omega, \mathcal{F}, \mathbb{P})$. 
To ensure the existence of $L^{2}$ bounded solutions to (4.1) we will impose some conditions on $\Lambda$. We assume that

(L2) i $\Lambda$ is the generator of a $C_{0}$ group $\left(\mathrm{e}^{\mathrm{i} t \Lambda}\right)_{t \in \mathbb{R}}$ of operators on $E$; by a corollary to the Hille-Yosida Theorem, for which see $\S 12.3$ of $[\mathbf{1 7}]$, this is equivalent to the resolvent of $\Lambda$ satisfying

$$
\left\|(\Lambda+\mathrm{i} \omega I)^{-n}\right\| \leqslant \frac{C_{\Lambda}}{\left(|\omega|-\alpha_{\Lambda}\right)^{n}}
$$

for some finite constants $C_{\Lambda}>0$ and $\alpha_{\Lambda} \geqslant 0$, all $n \in \mathbb{N}$ and all real $\omega$ such that $|\omega|>\alpha_{\Lambda} ;$

(L3) the resolvent of $\Lambda$ satisfies

$$
\left\|(\Lambda+\mathrm{i} \omega I)^{-1}\right\| \leqslant K_{\Lambda}
$$

for some finite constant $K_{\Lambda}>0$ and all real $\omega$; and

(L4) $\mathrm{i} \Lambda^{*}$ is the generator of a $C_{0}$ group $\left(\mathrm{e}^{\mathrm{i} t \Lambda^{*}}\right)_{t \in \mathbb{R}}$ of operators on $E^{*}$ (if $E$ is reflexive, this follows from (L2)).

For more information on these conditions, consult [8] or Chapters 11, 12 and 14 of [17]. Note that (L4), in the presence of (L2), is equivalent to the domain $\mathcal{D}\left(\Lambda^{*}\right)$ of $\Lambda^{*}$, the adjoint of $\Lambda$, being norm dense in $E^{*}$; without (L4) we only know it is weak-* dense. For details see $\S 1.4$ of $[\mathbf{8}]$ or Chapter 14 of $[\mathbf{1 7}]$.

We interpret equation (4.1) in the following way. The process $\left(\boldsymbol{B}_{t}\right)_{t \in \mathbb{R}}$ is required to be an $E$-valued $Q$-Wiener process. The process $\left(\boldsymbol{Z}_{t}\right)_{t \in \mathbb{R}}$ is required to be an $E$-valued, centred Gaussian, stationary stochastic process with almost surely Hölder continuous sample paths. As $\boldsymbol{Z}_{t}$ is stationary we may write $\operatorname{Cov}\left(\boldsymbol{Z}_{t}, \boldsymbol{Z}_{s}\right)=\Psi_{\boldsymbol{Z}}(t-s)$ for some function $\Psi_{\boldsymbol{Z}}$, called the autocovariance function of the process. Finally, we require that $\boldsymbol{B}_{t}$ and $\boldsymbol{Z}_{t}$ satisfy

$$
\xi^{*}\left(\boldsymbol{Z}_{t}-\boldsymbol{Z}_{s}\right)+\int_{s}^{t} \Lambda^{*}\left(\xi^{*}\right)\left(\boldsymbol{Z}_{u}\right) \mathrm{d} u=\xi^{*}\left(\boldsymbol{B}_{t}-\boldsymbol{B}_{s}\right)
$$

almost surely, for all $\xi^{*} \in \mathcal{D}\left(\Lambda^{*}\right)$ and $s<t$. Following [6] or Chapter 5 of [7] we call the pair $\left(\boldsymbol{B}_{t}, \boldsymbol{Z}_{t}\right)$ a weak solution of the Langevin equation.

Condition (L4), together with the separability of $E$ and the Hahn-Banach Theorem, implies there is a countable subset of $\mathcal{D}\left(\Lambda^{*}\right)$ which separates the points of $E$. This ensures that, if we know $\xi^{*}\left(\boldsymbol{B}_{t}\right)$ and $\xi^{*}\left(\boldsymbol{Z}_{t}\right)$ almost surely for all $\xi^{*} \in \mathcal{D}\left(\Lambda^{*}\right)$, the processes $\boldsymbol{B}_{t}$ and $\boldsymbol{Z}_{t}$ are almost surely determined.

Note we do not require that $\boldsymbol{Z}_{t}$ be adapted to the filtration induced by $\boldsymbol{B}_{t}$; we will, however, consider important circumstances in which this is the case.

\subsection{Spectral solutions of the Langevin equation}

This section states and proves an existence theorem for weak solutions of equation (4.1). Consider

$$
\boldsymbol{Z}_{t}=\frac{1}{\sqrt{2 \pi}} \int_{-\infty}^{\infty} \mathrm{e}^{\mathrm{i} \omega t}(\Lambda+\mathrm{i} \omega I)^{-1} \mathrm{~d} \tilde{\boldsymbol{B}}_{\omega}
$$


where $\tilde{\boldsymbol{B}}_{\omega}$ is a given $E$-valued $Q$-Wiener process defined on $(\Omega, \mathcal{F}, \mathbb{P})$. This formula is suggested by classical harmonic analysis. Consider also $\boldsymbol{B}_{t}$ defined by the condition $\boldsymbol{B}_{0}=0$ and, for $s<t$,

$$
\boldsymbol{B}_{t}-\boldsymbol{B}_{s}=\frac{1}{\sqrt{2 \pi}} \int_{-\infty}^{\infty} \frac{\mathrm{e}^{\mathrm{i} \omega t}-\mathrm{e}^{\mathrm{i} \omega s}}{\mathrm{i} \omega} \mathrm{d} \tilde{\boldsymbol{B}}_{\omega}
$$

All our stochastic integrals will be interpreted within the framework of Theorem 3.7.

Theorem 4.1. Assume that conditions (L1)-(L4) hold. The expression $\boldsymbol{Z}_{t}$ above

(a) converges as a stochastic integral for each $t \in \mathbb{R}$, defining an $E$-valued centred Gaussian process on $(\Omega, \mathcal{F}, \mathbb{P})$;

(b) is a stationary process with bounded autocovariance $\Psi_{Z}$ given by

$$
\Psi_{\boldsymbol{Z}}(u)=\frac{1}{2 \pi} \int_{-\infty}^{\infty} \mathrm{e}^{\mathrm{i} \omega u}(\Lambda+\mathrm{i} \omega I)^{-1} Q\left[(\bar{\Lambda}-\mathrm{i} \omega I)^{-1}\right]^{*} \mathrm{~d} \omega
$$

and

(c) has almost surely Hölder continuous sample paths, of exponent $\alpha$ for every $\alpha<\frac{1}{2}$.

The expression $\boldsymbol{B}_{t}$ above

(d) has increments $\boldsymbol{B}_{t}-\boldsymbol{B}_{s}$ which converge as stochastic integrals for each $s<t$, defining $E$-valued centred Gaussian random vectors on $(\Omega, \mathcal{F}, \mathbb{P})$; and

(e) is an $E$-valued $Q$-Wiener process defined on $(\Omega, \mathcal{F}, \mathbb{P})$.

Furthermore,

(f) the pair $\left(\boldsymbol{B}_{t}, \boldsymbol{Z}_{t}\right)$ is a weak solution of the E-valued Langevin equation (4.1).

Proof. (a) Assume $Q$ factors as $A \overline{A^{*}}$. Let us consider, for finite $a<b$,

$$
\boldsymbol{Z}_{t}^{(a, b)}=\frac{1}{\sqrt{2 \pi}} \int_{a}^{b} \mathrm{e}^{\mathrm{i} \omega t}(\Lambda+\mathrm{i} \omega I)^{-1} \mathrm{~d} \tilde{\boldsymbol{B}}_{\omega} .
$$

By Theorem 3.7 this expression will converge as a stochastic integral to a centred Gaussian element of $L^{2}(\Omega ; E)$ if the family $\left(A^{*}\left[\mathrm{e}^{\mathrm{i} \omega t}(\Lambda+\mathrm{i} \omega I)^{-1}\right]^{*}\right)_{a \leqslant \omega \leqslant b}$ is continuous in the $\pi_{2}$ norm. This in turn will follow if the family $\left(\mathrm{e}^{\mathrm{i} \omega t}(\Lambda+\mathrm{i} \omega I)^{-1}\right)_{a \leqslant \omega \leqslant b}$ is operator norm continuous, since $A^{*}$ is 2 -summing. But, for $a \leqslant p<q \leqslant b$,

$$
\begin{aligned}
\mathrm{e}^{\mathrm{i} q t}(\Lambda+\mathrm{i} q I)^{-1} & -\mathrm{e}^{\mathrm{i} p t}(\Lambda+\mathrm{i} p I)^{-1} \\
& =\left(\mathrm{e}^{\mathrm{i} q t}-\mathrm{e}^{\mathrm{i} p t}\right)(\Lambda+\mathrm{i} p I)^{-1}+\mathrm{e}^{\mathrm{i} q t}\left((\Lambda+\mathrm{i} q I)^{-1}-(\Lambda+\mathrm{i} p I)^{-1}\right) \\
& =\left(\mathrm{e}^{\mathrm{i} q t}-\mathrm{e}^{\mathrm{i} p t}\right)(\Lambda+\mathrm{i} p I)^{-1}-\mathrm{e}^{\mathrm{i} q t}(q-p)(\Lambda+\mathrm{i} p I)^{-1}(\Lambda+\mathrm{i} q I)^{-1}
\end{aligned}
$$

by the resolvent equation. We have $\left|\mathrm{e}^{\mathrm{i} q t}-\mathrm{e}^{\mathrm{i} p t}\right| \leqslant(q-p)|t|$; this and condition (L3) show that

$$
\left\|\mathrm{e}^{\mathrm{i} q t}(\Lambda+\mathrm{i} q I)^{-1}-\mathrm{e}^{\mathrm{i} p t}(\Lambda+\mathrm{i} p I)^{-1}\right\| \leqslant K_{\Lambda}\left(|t|+K_{\Lambda}\right)(q-p),
$$


which proves that $\left(\mathrm{e}^{\mathrm{i} \omega t}(\Lambda+\mathrm{i} \omega I)^{-1}\right)_{a \leqslant \omega \leqslant b}$ is continuous, indeed Hölder continuous, in operator norm, as required. We deduce that $\boldsymbol{Z}_{t}^{(a, b)}$ defines a centred Gaussian element of $L^{2}(\Omega ; E)$.

By Proposition 3.1 (b) the integral $\boldsymbol{Z}_{t}^{(a, b)}$ will converge in $L_{w}^{2}\left(\Omega ; E, \pi_{2}\right)$ as $a \downarrow-\infty$ and $b \uparrow \infty$ if, for any $\varepsilon>0$, there exists a positive finite $N(\varepsilon)$ such that $\left\|\boldsymbol{Z}_{t}^{(m, n)}\right\|_{\pi_{2}}<\varepsilon$ for all $n>m \geqslant N(\varepsilon)$. By Theorem 3.7 and condition (L2) we have

$$
\begin{aligned}
\left\|\boldsymbol{Z}_{t}^{(m, n)}\right\|_{\pi_{2}}^{2} & =\left\|\frac{1}{\sqrt{2 \pi}} \int_{m}^{n} \mathrm{e}^{\mathrm{i} \omega t}(\Lambda+\mathrm{i} \omega I)^{-1} \mathrm{~d} \tilde{\boldsymbol{B}}_{\omega}\right\|_{\pi_{2}}^{2} \\
& \leqslant \frac{1}{2 \pi} \int_{m}^{n} \pi_{2}\left(\left((\Lambda+\mathrm{i} \omega I)^{-1} A\right)^{*}\right)^{2} \mathrm{~d} \omega \\
& \leqslant \frac{\pi_{2}\left(A^{*}\right)^{2}}{2 \pi} \int_{m}^{n}\left\|(\Lambda+\mathrm{i} \omega I)^{-1}\right\|^{2} \mathrm{~d} \omega \\
& <\frac{C_{\Lambda}^{2} \pi_{2}\left(A^{*}\right)^{2}}{2 \pi\left(m-\alpha_{\Lambda}\right)}
\end{aligned}
$$

for all $n>m>\alpha_{\Lambda}$, as required. We deduce that $\boldsymbol{Z}_{t}=\lim _{a \downarrow-\infty, b \uparrow \infty} \boldsymbol{Z}_{t}^{(a, b)}$ converges in $L_{w}^{2}\left(\Omega ; E, \pi_{2}\right)$ to a centred Gaussian element of $L^{2}(\Omega ; E)$.

(b) Fix $s<t$. For finite $a<b$, using the notation $\boldsymbol{Z}_{t}^{(a, b)}$ from part (a),

$$
\begin{aligned}
\operatorname{Cov}\left(\boldsymbol{Z}_{t}^{(a, b)}, \boldsymbol{Z}_{s}^{(a, b)}\right) & =\frac{1}{2 \pi} \operatorname{Cov}\left(\int_{a}^{b} \mathrm{e}^{\mathrm{i} \omega t}(\Lambda+\mathrm{i} \omega I)^{-1} \mathrm{~d} \tilde{\boldsymbol{B}}_{\omega}, \int_{a}^{b} \mathrm{e}^{\mathrm{i} \omega^{\prime} s}\left(\Lambda+\mathrm{i} \omega^{\prime} I\right)^{-1} \mathrm{~d} \tilde{\boldsymbol{B}}_{\omega^{\prime}}\right) \\
& =\frac{1}{2 \pi} \lim _{L^{2}} \operatorname{Cov}\left(\sum_{j=0}^{r-1} \mathrm{e}^{\mathrm{i} \omega_{j} t}\left(\Lambda+\mathrm{i} \omega_{j} I\right)^{-1}\left(\tilde{\boldsymbol{B}}_{\omega_{j+1}}-\tilde{\boldsymbol{B}}_{\omega_{j}}\right),\right. \\
& \left.\sum_{k=0}^{r-1} \mathrm{e}^{\mathrm{i} \omega_{k}^{\prime} s}\left(\Lambda+\mathrm{i} \omega_{k}^{\prime} I\right)^{-1}\left(\tilde{\boldsymbol{B}}_{\omega_{k+1}^{\prime}}-\tilde{\boldsymbol{B}}_{\omega_{k}^{\prime}}\right)\right) \\
& =\frac{1}{2 \pi} \lim _{L^{2}} \sum_{j=0}^{r-1} \mathrm{e}^{\mathrm{i} \omega_{j}(t-s)} \operatorname{Var}\left(\left(\Lambda+\mathrm{i} \omega_{j} I\right)^{-1}\left(\tilde{\boldsymbol{B}}_{\omega_{j+1}}-\tilde{\boldsymbol{B}}_{\omega_{j}}\right)\right),
\end{aligned}
$$

where the $L^{2}$ limit is taken to mean the limit in $L_{w}^{2}\left(\Omega ; E, \pi_{2}\right)$ over refinements of appropriate partitions $a=\omega_{0}=\omega_{0}^{\prime}<\omega_{1}=\omega_{1}^{\prime}<\cdots<\omega_{r}=\omega_{r}^{\prime}=b$ of $[a, b]$. By Proposition 3.5 (ii) we see

$$
\begin{aligned}
\operatorname{Cov}\left(\boldsymbol{Z}_{t}^{(a, b)}, \boldsymbol{Z}_{s}^{(a, b)}\right) & =\frac{1}{2 \pi} \lim _{L^{2}} \sum_{j=0}^{r-1} \mathrm{e}^{\mathrm{i} \omega_{j}(t-s)}\left(\Lambda+\mathrm{i} \omega_{j} I\right)^{-1} Q\left[\left(\bar{\Lambda}-\mathrm{i} \omega_{j} I\right)^{-1}\right]^{*}\left(\omega_{j+1}-\omega_{j}\right) \\
& =\frac{1}{2 \pi} \int_{a}^{b} \mathrm{e}^{\mathrm{i} \omega(t-s)}(\Lambda+\mathrm{i} \omega I)^{-1} Q\left[(\bar{\Lambda}-\mathrm{i} \omega I)^{-1}\right]^{*} \mathrm{~d} \omega
\end{aligned}
$$

Letting $a \downarrow-\infty$ and $b \uparrow \infty$ yields the required formula for the autocovariance; it is clear that $\boldsymbol{Z}_{t}$ is stationary.

(c) We have, for $s<t$,

$$
\boldsymbol{Z}_{t}-\boldsymbol{Z}_{s}=\frac{1}{\sqrt{2 \pi}} \int_{-\infty}^{\infty}\left(\mathrm{e}^{\mathrm{i} \omega t}-\mathrm{e}^{\mathrm{i} \omega s}\right)(\Lambda+\mathrm{i} \omega I)^{-1} \mathrm{~d} \tilde{\boldsymbol{B}}_{\omega},
$$


which, applying Theorem 3.7, yields

$$
\left\|\boldsymbol{Z}_{t}-\boldsymbol{Z}_{s}\right\|_{\pi_{2}}^{2} \leqslant \frac{\pi_{2}\left(A^{*}\right)^{2}}{2 \pi} \int_{-\infty}^{\infty}\left|\mathrm{e}^{\mathrm{i} \omega t}-\mathrm{e}^{\mathrm{i} \omega s}\right|^{2}\left\|(\Lambda+\mathrm{i} \omega I)^{-1}\right\|^{2} \mathrm{~d} \omega
$$

We now split the integral into separate parts with ranges $|\omega|<2 \alpha_{\Lambda}$ and $|\omega| \geqslant 2 \alpha_{\Lambda}$. Note that

$$
\left|\mathrm{e}^{\mathrm{i} \omega t}-\mathrm{e}^{\mathrm{i} \omega s}\right| \leqslant \min (2,|\omega|(t-s)) \leqslant \sqrt{2}|\omega|^{1 / 2}(t-s)^{1 / 2}
$$

and, for $|\omega| \geqslant 2 \alpha_{\Lambda}$, that $\left(|\omega|-\alpha_{\Lambda}\right)^{-1} \leqslant 2|\omega|^{-1}$. Applying conditions (L2) and (L3) gives

$$
\begin{aligned}
\left\|\boldsymbol{Z}_{t}-\boldsymbol{Z}_{s}\right\|_{\pi_{2}}^{2} & \leqslant \frac{K_{\Lambda}^{2} \pi_{2}\left(A^{*}\right)^{2}(t-s)}{\pi} \int_{|\omega|<2 \alpha_{\Lambda}}|\omega| \mathrm{d} \omega \\
& \quad+\frac{4 C_{\Lambda}^{2} \pi_{2}\left(A^{*}\right)^{2}}{2 \pi} \int_{|\omega| \geqslant 2 \alpha_{\Lambda}}\left|\frac{\mathrm{e}^{\mathrm{i} \omega t}-\mathrm{e}^{\mathrm{i} \omega s}}{\mathrm{i} \omega}\right|^{2} \mathrm{~d} \omega \\
& \leqslant 4\left(\frac{\alpha_{\Lambda}^{2} K_{\Lambda}^{2}}{\pi}+C_{\Lambda}^{2}\right) \pi_{2}\left(A^{*}\right)^{2}(t-s)
\end{aligned}
$$

by Plancherel's Theorem applied to the indicator function of $[s, t]$, as required. This expression shows that the map $t \mapsto \boldsymbol{Z}_{t}$ is Hölder continuous as a function $\mathbb{R} \rightarrow$ $L_{w}^{2}\left(\Omega ; E, \pi_{2}\right)$ with exponent $\frac{1}{2}$. The fact that $\boldsymbol{Z}_{t}$ is Gaussian enables us to now apply Kolmogorov's Continuity Lemma in its vector-valued form to deduce the existence of a version of $\boldsymbol{Z}_{t}$ with almost surely Hölder continuous sample paths of exponent $\alpha$ for every $\alpha<\frac{1}{2}$ (see pp. 59-61 of [33] for details).

(d) Fix $s<t$. Keeping the notation of part (a) we define $\boldsymbol{B}_{t}^{(a, b)}-\boldsymbol{B}_{s}^{(a, b)}$, for finite $a<b$, in the same manner as we defined $\boldsymbol{Z}_{t}^{(a, b)}$. By Theorem 3.7 this will converge as a stochastic integral to a centred Gaussian element of $L^{2}(\Omega ; E)$ if the family

$$
\left(\frac{\mathrm{e}^{\mathrm{i} \omega t}-\mathrm{e}^{\mathrm{i} \omega s}}{\mathrm{i} \omega}\right)_{a \leqslant \omega \leqslant b}
$$

is continuous. But, for $a \leqslant p<q \leqslant b$,

$$
\begin{aligned}
\left|\frac{\mathrm{e}^{\mathrm{i} q t}-\mathrm{e}^{\mathrm{i} q s}}{\mathrm{i} q}-\frac{\mathrm{e}^{\mathrm{i} p t}-\mathrm{e}^{\mathrm{i} p s}}{\mathrm{i} p}\right| & =\left|\int_{s}^{t}\left(\mathrm{e}^{\mathrm{i} q u}-\mathrm{e}^{\mathrm{i} p u}\right) \mathrm{d} u\right| \\
& \leqslant\left(\int_{s}^{t}|u| \mathrm{d} u\right)(q-p),
\end{aligned}
$$

and so we have continuity, indeed Hölder continuity, as required. Thus the stochastic integral $\boldsymbol{B}_{t}^{(a, b)}-\boldsymbol{B}_{s}^{(a, b)}$ defines a centred Gaussian element of $L^{2}(\Omega ; E)$.

To consider the case when $a \downarrow-\infty$ and $b \uparrow \infty$ we note by Theorem 3.7 that

$$
\left\|\boldsymbol{B}_{t}^{(a, b)}-\boldsymbol{B}_{s}^{(a, b)}\right\|_{\pi_{2}}^{2} \leqslant \frac{\pi_{2}\left(A^{*}\right)^{2}}{2 \pi} \int_{a}^{b}\left|\frac{\mathrm{e}^{\mathrm{i} \omega t}-\mathrm{e}^{\mathrm{i} \omega s}}{\mathrm{i} \omega}\right|^{2} \mathrm{~d} \omega .
$$

By Plancherel's Theorem, applied to the indicator function of $[s, t]$, this integral increases to $\pi_{2}\left(A^{*}\right)^{2}(t-s)$ as $a \downarrow-\infty$ and $b \uparrow \infty$. It follows that, for any $\varepsilon>0$, there exists a positive finite $N(\varepsilon)$ such that $\left\|\boldsymbol{B}_{t}^{(m, n)}-\boldsymbol{B}_{s}^{(m, n)}\right\|_{\pi_{2}}<\varepsilon$ for all $n>m \geqslant N(\varepsilon)$; consequently, 
by Proposition 3.1 (b), the stochastic integral $\boldsymbol{B}_{t}-\boldsymbol{B}_{s}=\lim _{a \downarrow-\infty, b \uparrow \infty}\left(\boldsymbol{B}_{t}^{(a, b)}-\boldsymbol{B}_{s}^{(a, b)}\right)$ converges in $L_{w}^{2}\left(\Omega ; E, \pi_{2}\right)$ to a centred Gaussian element of $L^{2}(\Omega ; E)$.

(e) For $s<t$, by part (d) above,

$$
\left\|\boldsymbol{B}_{t}-\boldsymbol{B}_{s}\right\|_{\pi_{2}}^{2} \leqslant \pi_{2}\left(A^{*}\right)^{2}(t-s) .
$$

We may therefore apply Kolmogorov's Continuity Lemma in the same manner as part (c) to deduce almost sure sample path continuity.

By a calculation similar to that of part (b),

$$
\begin{aligned}
\operatorname{Var}\left(\boldsymbol{B}_{t}-\boldsymbol{B}_{s}\right) & =\frac{1}{2 \pi} \int_{-\infty}^{\infty}\left|\frac{\mathrm{e}^{\mathrm{i} \omega t}-\mathrm{e}^{\mathrm{i} \omega s}}{\mathrm{i} \omega}\right|^{2} \mathrm{~d} \omega Q \\
& =(t-s) Q
\end{aligned}
$$

by Plancherel's Theorem applied to the indicator function of $[s, t]$; also, for $s<t<u<v$,

$$
\begin{aligned}
\operatorname{Cov}\left(\boldsymbol{B}_{t}-\boldsymbol{B}_{s}, \boldsymbol{B}_{v}-\boldsymbol{B}_{u}\right) & =\frac{1}{2 \pi} \int_{-\infty}^{\infty} \frac{\left(\mathrm{e}^{\mathrm{i} \omega t}-\mathrm{e}^{\mathrm{i} \omega s}\right)\left(\mathrm{e}^{-\mathrm{i} \omega v}-e^{-\mathrm{i} \omega u}\right)}{|\mathrm{i} \omega|^{2}} \mathrm{~d} \omega Q \\
& =0
\end{aligned}
$$

again by Plancherel's Theorem, this time applied to the indicator functions of the disjoint intervals $[s, t]$ and $[u, v]$. We deduce that $\boldsymbol{B}_{t}$ is a $Q$-Wiener process as required.

(f) For $\xi^{*} \in \mathcal{D}\left(\Lambda^{*}\right)$ and $s<t$ we calculate

$$
\begin{aligned}
& \xi^{*}\left(\boldsymbol{Z}_{t}-\boldsymbol{Z}_{s}\right)+\int_{s}^{t} \Lambda^{*}\left(\xi^{*}\right)\left(\boldsymbol{Z}_{u}\right) \mathrm{d} u \\
&= \frac{1}{\sqrt{2 \pi}} \xi^{*}\left(\int_{-\infty}^{\infty}\left(\mathrm{e}^{\mathrm{i} \omega t}-\mathrm{e}^{\mathrm{i} \omega s}\right)(\Lambda+\mathrm{i} \omega I)^{-1} \mathrm{~d} \tilde{\boldsymbol{B}}_{\omega}\right) \\
& \quad+\frac{1}{\sqrt{2 \pi}} \int_{s}^{t} \Lambda^{*}\left(\xi^{*}\right)\left(\int_{-\infty}^{\infty} \mathrm{e}^{\mathrm{i} \omega u}(\Lambda+\mathrm{i} \omega I)^{-1} \mathrm{~d} \tilde{\boldsymbol{B}}_{\omega}\right) \mathrm{d} u \\
&=\frac{1}{\sqrt{2 \pi}} \int_{-\infty}^{\infty}\left(\frac{\mathrm{e}^{\mathrm{i} \omega t}-\mathrm{e}^{\mathrm{i} \omega s}}{\mathrm{i} \omega}\right) \mathrm{i} \omega \xi^{*}(\Lambda+\mathrm{i} \omega I)^{-1} \mathrm{~d} \tilde{\boldsymbol{B}}_{\omega} \\
& \quad+\frac{1}{\sqrt{2 \pi}} \int_{-\infty}^{\infty}\left(\int_{s}^{t} \mathrm{e}^{\mathrm{i} \omega u} \mathrm{~d} u\right) \Lambda^{*}\left(\xi^{*}\right)(\Lambda+\mathrm{i} \omega I)^{-1} \mathrm{~d} \tilde{\boldsymbol{B}}_{\omega},
\end{aligned}
$$

where the change in the order of integration in the second integral is justified by the almost sure Hölder continuity of the sample paths of $\boldsymbol{Z}_{u}$ and the fact that, for each $u$, $\boldsymbol{Z}_{u}$ is the limit in $L_{w}^{2}\left(\Omega ; E, \pi_{2}\right)$ of a sequence of Riemann sums. This gives

$$
\begin{aligned}
\xi^{*}\left(\boldsymbol{Z}_{t}-\boldsymbol{Z}_{s}\right) & +\int_{s}^{t} \Lambda^{*}\left(\xi^{*}\right)\left(\boldsymbol{Z}_{u}\right) \mathrm{d} u \\
& =\frac{1}{\sqrt{2 \pi}} \int_{-\infty}^{\infty}\left(\frac{\mathrm{e}^{\mathrm{i} \omega t}-\mathrm{e}^{\mathrm{i} \omega s}}{\mathrm{i} \omega}\right)\left(\mathrm{i} \omega \xi^{*}+\Lambda^{*}\left(\xi^{*}\right)\right)(\Lambda+\mathrm{i} \omega I)^{-1} \mathrm{~d} \tilde{\boldsymbol{B}}_{\omega} \\
& =\frac{1}{\sqrt{2 \pi}} \int_{-\infty}^{\infty}\left(\frac{\mathrm{e}^{\mathrm{i} \omega t}-\mathrm{e}^{\mathrm{i} \omega s}}{\mathrm{i} \omega}\right)\left(\mathrm{i} \omega \xi^{*}+\xi^{*} \Lambda\right)(\Lambda+\mathrm{i} \omega I)^{-1} \mathrm{~d} \tilde{\boldsymbol{B}}_{\omega} ;
\end{aligned}
$$


we know that $\Lambda^{*}\left(\xi^{*}\right)=\xi^{*} \Lambda$ because the range of the resolvent satisfies

$$
(\Lambda+\mathrm{i} \omega I)^{-1}(E) \subseteq \mathcal{D}(\Lambda)
$$

for all $\omega \in \mathbb{R}$. Thus

$$
\begin{aligned}
\xi^{*}\left(\boldsymbol{Z}_{t}-\boldsymbol{Z}_{s}\right)+\int_{s}^{t} \Lambda^{*}\left(\xi^{*}\right)\left(\boldsymbol{Z}_{u}\right) \mathrm{d} u & =\frac{1}{\sqrt{2 \pi}} \int_{-\infty}^{\infty}\left(\frac{\mathrm{e}^{\mathrm{i} \omega t}-\mathrm{e}^{\mathrm{i} \omega s}}{\mathrm{i} \omega}\right) \xi^{*}\left(\mathrm{~d} \tilde{\boldsymbol{B}}_{\omega}\right) \\
& =\frac{1}{\sqrt{2 \pi}} \xi^{*}\left(\int_{-\infty}^{\infty} \frac{\mathrm{e}^{\mathrm{i} \omega t}-\mathrm{e}^{\mathrm{i} \omega s}}{\mathrm{i} \omega} \mathrm{d} \tilde{\boldsymbol{B}}_{\omega}\right) \\
& =\xi^{*}\left(\boldsymbol{B}_{t}-\boldsymbol{B}_{s}\right),
\end{aligned}
$$

which is justified by the convergence in $L_{w}^{2}\left(\Omega ; E, \pi_{2}\right)$ of the stochastic integral $\boldsymbol{B}_{t}-\boldsymbol{B}_{s}$. We deduce that $\left(\boldsymbol{B}_{t}, \boldsymbol{Z}_{t}\right)$ is a weak solution of the $E$-valued Langevin equation (4.1).

The proof of Theorem 4.1 (f) naturally generalizes the corresponding proof in the scalar-valued case (for details of this, see Chapter XI, $\S 10$ of [11]).

\subsection{A sufficient condition for adaptedness and uniqueness}

We now consider a condition on $\Lambda$ under which $\boldsymbol{Z}_{t}$ is adapted to the filtration induced by $\boldsymbol{B}_{t}$, and $\boldsymbol{Z}_{t}$ is unique in distribution. The following corollary to Theorem 4.1 mirrors results in Itô's paper [18].

Corollary 4.2. Let $\left(\boldsymbol{B}_{t}, \boldsymbol{Z}_{t}\right)$ be a weak solution of the $E$-valued Langevin equation (4.1). Assume, as well as (L1)-(L4), that the operator $(-\Lambda)$ generates a $C_{0}$ semigroup $\left(\mathrm{e}^{-\Lambda t}\right)_{t \geqslant 0}$ of exponential norm decay. Then the process $\boldsymbol{Z}_{t}$ is adapted to the filtration induced by $\boldsymbol{B}_{t}$; furthermore, if $\left(\boldsymbol{B}_{t}^{\prime}, \boldsymbol{Z}_{t}^{\prime}\right)$ is also a weak solution, then $\boldsymbol{Z}_{t}^{\prime}$ is identical in distribution to $\boldsymbol{Z}_{t}$.

Proof. Firstly let $\left(\boldsymbol{B}_{t}, \boldsymbol{Z}_{t}\right)$ and $\left(\boldsymbol{B}_{t}, \boldsymbol{Z}_{t}^{\prime}\right)$ be weak solutions of the $E$-valued Langevin equation. Setting $\Theta_{t}=\boldsymbol{Z}_{t}^{\prime}-\boldsymbol{Z}_{t}$ we see that $\Theta_{t}$ is an $E$-valued, centred Gaussian, stationary stochastic process with almost surely Hölder continuous sample paths defined on $(\Omega, \mathcal{F}, \mathbb{P})$ which satisfies

$$
\xi^{*}\left(\Theta_{t}-\Theta_{s}\right)+\int_{s}^{t} \Lambda^{*}\left(\xi^{*}\right)\left(\Theta_{u}\right) \mathrm{d} u=0
$$

almost surely, for all $\xi^{*} \in \mathcal{D}\left(\Lambda^{*}\right)$ and $s<t$. Itô's paper [18] now shows, for $s<t$,

$$
\Theta_{t}=\mathrm{e}^{-\Lambda(t-s)} \Theta_{s}
$$

The condition that $\left(\mathrm{e}^{-\Lambda t}\right)_{t \geqslant 0}$ is of exponential norm decay, together with the requirement that $\Theta_{t}$ be stationary, now implies $\Theta_{t}=0$ almost surely, we simply let $t$ tend to infinity in $(4.27)$. 
Now, by [18], if $\boldsymbol{B}_{t}$ is any $E$-valued $Q$-Wiener process, then the process

$$
\boldsymbol{Z}_{t}=\int_{-\infty}^{t} \mathrm{e}^{-\Lambda(t-u)} \mathrm{d} \boldsymbol{B}_{u}
$$

is such that $\left(\boldsymbol{B}_{t}, \boldsymbol{Z}_{t}\right)$ is a weak solution of the $E$-valued Langevin equation.

Combining these results we see that, given $\boldsymbol{B}_{t}$, the process $\boldsymbol{Z}_{t}$ is almost surely unique and is given almost surely by the stochastic integral (4.28).

The stochastic integral (4.28) shows that $\boldsymbol{Z}_{t}$ is adapted to the filtration induced by $\boldsymbol{B}_{t}$. Furthermore, we see that, whenever $\left(\boldsymbol{B}_{t}, \boldsymbol{Z}_{t}\right)$ and $\left(\boldsymbol{B}_{t}^{\prime}, \boldsymbol{Z}_{t}^{\prime}\right)$ are weak solutions, $\boldsymbol{Z}_{t}$ must be expressible almost surely in the form (4.28) as a stochastic integral with respect to $\boldsymbol{B}_{t}$, and $\boldsymbol{Z}_{t}^{\prime}$ must be expressible almost surely in the form (4.28) as a stochastic integral with respect to $\boldsymbol{B}_{t}^{\prime}$. We deduce, as $\boldsymbol{B}_{t}$ and $\boldsymbol{B}_{t}^{\prime}$ have the same distribution, that $\boldsymbol{Z}_{t}$ and $\boldsymbol{Z}_{t}^{\prime}$ have the same distribution.

Remark 4.3. We pose two questions. Under what precise conditions is the OrnsteinUhlenbeck process $\boldsymbol{Z}_{t}$ unique in distribution? Under what precise conditions is $\boldsymbol{Z}_{t}$ adapted to the filtration induced by the $Q$-Wiener process $\boldsymbol{B}_{t}$ ? Corollary 4.2 provides a sufficient condition for both adaptedness and uniqueness.

\subsection{Examples}

Let $1<p<\infty$ and $\varepsilon>0$. Define

$$
E=\left\{f \in L^{p}(\mathbb{R}): \hat{f}(\zeta)=0 \text { for all }|\zeta| \leqslant \varepsilon\right\},
$$

where $\hat{f}$ denotes the Fourier transform of $f$. The continuity of the Riesz projection on $L^{p}(\mathbb{R})\left[\mathbf{2 3}\right.$, Section V.B] shows that $E$ is a closed complemented subspace of $L^{p}(\mathbb{R})$; it is therefore a reflexive Banach space.

We consider various possibilities for $\Lambda$, each defined via Fourier multipliers.

(i) $\left.\widehat{\left(\mathrm{e}^{\mathrm{i} t \Lambda} f\right.}\right)(\zeta)=\mathrm{e}^{\mathrm{i} t \zeta} \hat{f}(\zeta)$, so $\left(\mathrm{e}^{\mathrm{i} t \Lambda} f\right)(x)=f(x+t)$ for all $x \in \mathbb{R}$ and $\left(\mathrm{e}^{\mathrm{i} t \Lambda}\right)_{t \in \mathbb{R}}$ is a $C_{0}$ group of translation operators on $L^{p}(\mathbb{R})$ with $\Lambda=-\mathrm{i}(\mathrm{d} / \mathrm{d} x)$.

(ii) $\widehat{\left(\mathrm{e}^{\mathrm{i} t \Lambda f}\right)}(\zeta)=\mathrm{e}^{\mathrm{i} t|\zeta|} \hat{f}(\zeta)$, so $\left(\mathrm{e}^{\mathrm{i} t \Lambda} f\right)(x)=\left(\mathcal{R}_{+} f\right)(x+t)+\left(\mathcal{R}_{-} f\right)(x-t)$ for all $x \in \mathbb{R}$, where $\mathcal{R}_{+}$and $\mathcal{R}_{-}$denote the positive and negative Riesz projections.

(iii) $\left.\widehat{\left(\mathrm{e}^{\mathrm{i} t \Lambda} f\right.}\right)(\zeta)=\mathrm{e}^{\mathrm{i} t \log |\zeta|} \hat{f}(\zeta)$, implying $\mathrm{e}^{\mathrm{i} t \Lambda}=\Delta^{\mathrm{i} t}$, where $\Delta=-\mathrm{d}^{2} / \mathrm{d} x^{2}$ is the Laplace operator. The Laplacian is essentially self-adjoint on $C_{c}^{\infty}(\mathbb{R})$ (see Chapter 4 of $[8]$ for details), which enables us to define its imaginary powers by Fourier multipliers as described. These imaginary powers $\left(\Delta^{\mathrm{i} t}\right)_{t \in \mathbb{R}}$ form a $C_{0}$ group on $L^{p}(\mathbb{R})$ of polynomial growth (see [16] for details).

All the $\left(\mathrm{e}^{\mathrm{i} t \Lambda}\right)_{t \in \mathbb{R}}$ considered here restrict to $E$, yielding $C_{0}$ groups. The condition on each $f$ in $E$ that $\hat{f}(\zeta)=0$ for all $|\zeta| \leqslant \varepsilon$ implies, in all cases, that the resolvent of $\Lambda$ is bounded on the imaginary axis; conditions (L2)-(L4) are therefore satisfied. In addition, neither $\Lambda$ nor $(-\Lambda)$ generate $C_{0}$ semigroups of exponential norm decay. 
Thus, given any such $\Lambda$ and any $Q$ which is the variance of some centred Gaussian random vector in $E$, the $E$-valued Langevin equation (4.1) associated to $\Lambda$ and $Q$ has a weak solution as described in Theorem 4.1.

Acknowledgements. While writing this paper the author was supported by EPSRC research studentship no. 96006028; it forms part of his $\mathrm{PhD}$ thesis [15]. I thank my supervisor Dr G. Blower for his advice, enthusiasm and suggestions. I am indebted to the referee, whose comments and references have been extremely helpful. I have also enjoyed many illuminating conversations with Dr P. A. Haworth.

\section{References}

1. A. Blanc-Lapierre and R. Fortet, Sur la décomposition spectrale des fonctions aléatoires stationaires d'ordre deux, C. R. Acad. Sci. Paris 222 (1946), 467-468.

2. Z. BrZeźNIAK, Stochastic partial differential equations in $M$-type 2 Banach spaces, Potential Analysis 4 (1995), 1-45.

3. Z. BRzeźniaK AND S. Peszat, Space-time continuous solutions to SPDEs driven by a homogeneous Wiener process, Studia Math. 137 (1999), 261-299.

4. Z. Brzeźniak and J. M. A. M. van NeERven, Stochastic convolution in separable Banach spaces and the stochastic linear Cauchy problem, Studia Math. 143 (2000), 4374 .

5. Z. CiesIELSKI, Hölder conditions for realizations of Gaussian processes, Trans. Am. Math. Soc. 99 (1961), 403-413.

6. G. Da Prato And J. ZabCZYK, Stochastic equations in infinite dimensions, Encyclopedia of Mathematics and its Applications, vol. 44 (Cambridge University Press, 1992).

7. G. Da Prato AND J. ZABCZYK, Ergodicity for infinite dimensional systems, London Mathematical Society Lecture Note Series, no. 229 (Cambridge University Press, 1996).

8. E. B. DAviES, One-parameter semigroups, London Mathematical Society Monographs, no. 15 (Academic, 1980).

9. J. Diestel And J. J. Uhl JR, Vector measures, Mathematical Surveys, no. 15 (American Mathematical Society, Providence, RI, 1977).

10. J. Diestel, H. Jarchow And A. Tonge, Absolutely summing operators, Cambridge Studies in Advanced Mathematics, no. 43 (Cambridge University Press, 1995).

11. J. L. Doob, Stochastic processes (Wiley, 1953).

12. X. Fernique, Intégrabilité des vecteurs gaussiens, C. R. Acad. Sci. Paris Sér. A $\mathbf{2 7 0}$ (1970), 1698-1699.

13. T. Figiel and N. Tomczak-Jaegermann, Projections onto Hilbertian subspaces of Banach spaces, Israel J. Math. 33 (1979), 155-171.

14. G. R. Grimmett And D. R. Stirzaker, Probability and random processes, 2nd edn (Oxford University Press, 1992).

15. J. S. Groves, A study of stochastic processes in Banach spaces, PhD thesis, University of Lancaster (2000).

16. S. Guerre-Delabrieve, Some remarks on complex powers of $(-\Delta)$ and UMD spaces, Illinois J. Math. 35 (1991), 401-407.

17. E. Hille AND R. S. PhilliPs, Functional analysis and semigroups, revised edn, American Mathematical Society Colloquium Publications, vol. 31 (American Mathematical Society, Providence, RI, 1957).

18. K. ITô, Infinite dimensional Ornstein-Uhlenbeck processes, in Stochastic Analysis: Proc. Taniguchi International Symp. on Stochastic Analysis, Katata and Kyoto, 1982 (ed. K. Itô), North-Holland Mathematical Library, vol. 32, pp. 197-224 (North-Holland, Amsterdam, 1984). 
19. K. Itô AND M. Nisio, On the convergence of sums of independent Banach space valued random variables, Osaka J. Math. 5 (1968), 35-48.

20. A. Y. Khinchin, Korrelationstheorie der stationären stochastischen Prozesse, Math. Annln 109 (1934), 604-615.

21. A. N. Kolmogorov, Statistical theory of oscillations with continuous spectrum, in Collected papers on the 30th anniversary of the Great October Socialist Revolution, vol. 1, pp. 242-252 (Akademii Nauk SSSR, Moscow and Leningrad, 1947).

22. T. Kolsrud, Gaussian random fields, infinite dimensional Ornstein-Uhlenbeck processes and symmetric Markov processes, Acta Appl. Math. 12 (1988), 237-263.

23. P. Koosis, Introduction to $H_{p}$ spaces, 2nd edn, Cambridge Tracts in Mathematics, no. 115 (Cambridge University Press, 1998).

24. S. KWAPIEŃ AND W. A. WOYCZYŃSKI, Random series and stochastic integrals: single and multiple, Probability and its Applications (Birkhäuser, Boston, 1992).

25. H. J. LAndau And L. A. Shepp, On the supremum of a Gaussian process, Sankhyā A 32 (1970), 369-378.

26. M. Ledoux And M. Talagrand, Probability in Banach spaces, Ergebnisse der Mathematik und ihrer Grenzgebiete, 3 Folge, vol. 23 (Springer, 1991).

27. V. Linde, V. I. Tarieladze and S. A. Čbanjan, Characterization of certain classes of Banach spaces by properties of Gaussian measures, Teor. Veroyatnost. Primenen. 25 (1980), 162-167.

28. P. MASANI, Dilations as propagators of Hilbertian varieties, SIAM J. Math. Analysis 9 (1978), 414-456.

29. P. Masani, Measurability and Pettis integration in Hilbert spaces, J. Reine Angew. Math. 297 (1978), 92-135.

30. A. Millet And W. Smoleński, On the continuity of Ornstein-Uhlenbeck processes in infinite dimensions, Prob. Theory Relat. Fields 92 (1992), 529-547.

31. G. PISIER, Factorization of linear operators and geometry of Banach spaces, Regional Conference Series in Mathematics, no. 60 (American Mathematical Society, Providence, RI, 1986).

32. G. PISIER, Factorization of operator valued analytic functions, Adv. Math. 93 (1992), 61-125.

33. L. C. G. Rogers And D. Williams, Diffusions, Markov processes and martingales, I, Foundations, 2nd edn, Wiley Series in Probability and Mathematical Statistics (Wiley, 1994).

34. D. W. Stroock, Probability theory: an analytic view (Cambridge University Press, 1993).

35. G. E. Uhlenbeck And L. S. Ornstein, On the theory of the Brownian motion, Phys. Rev. B 36 (1930), 823-841.

36. J. M. A. M. VAN NeERven, Nonsymmetric Ornstein-Uhlenbeck semigroups in Banach spaces, J. Funct. Analysis 155 (1998), 495-535. 